TRANSACTIONS OF THE

AMERICAN MATHEMATICAL SOCIETY

Volume 359, Number 8, August 2007, Pages 3859-3885

S 0002-9947(07)04128-1

Article electronically published on March 7, 2007

\title{
ALGEBRAIC HYPERGEOMETRIC TRANSFORMATIONS OF MODULAR ORIGIN
}

\author{
ROBERT S. MAIER
}

\begin{abstract}
It is shown that Ramanujan's cubic transformation of the Gauss hypergeometric function ${ }_{2} F_{1}$ arises from a relation between modular curves, namely the covering of $X_{0}(3)$ by $X_{0}(9)$. In general, when $2 \leqslant N \leqslant 7$, the $N$-fold cover of $X_{0}(N)$ by $X_{0}\left(N^{2}\right)$ gives rise to an algebraic hypergeometric transformation. The $N=2,3,4$ transformations are arithmetic-geometric mean iterations, but the $N=5,6,7$ transformations are new. In the final two cases the change of variables is not parametrized by rational functions, since $X_{0}(6), X_{0}(7)$ are of genus 1 . Since their quotients $X_{0}^{+}(6), X_{0}^{+}(7)$ under the Fricke involution (an Atkin-Lehner involution) are of genus 0 , the parametrization is by two-valued algebraic functions. The resulting hypergeometric transformations are closely related to the two-valued modular equations of Fricke and H. Cohn.
\end{abstract}

\section{INTRODUCTION}

Identifiable functions that satisfy functional equations of high degree are rare flowers. For this reason, much attention has been paid to Ramanujan's parametrized cubic transformation

$$
{ }_{2} F_{1}\left(\frac{1}{3}, \frac{2}{3} ; 1 ; 1-\left(\frac{1-x}{1+2 x}\right)^{3}\right)=(1+2 x){ }_{2} F_{1}\left(\frac{1}{3}, \frac{2}{3} ; 1 ; x^{3}\right)
$$

of a particular case of the Gauß hypergeometric function ${ }_{2} F_{1}(\alpha, \beta ; \gamma ; \cdot)$, namely ${ }_{2} F_{1}\left(\frac{1}{3}, \frac{2}{3} ; 1 ; \cdot\right)$. In Ramanujan's theory of elliptic integrals in signature 3 , the functional equation (1.1) appears as the cubic arithmetic-geometric mean iteration [3]. The first published proof, by the Borweins [5], relied on the existence of an action of the modular group $\Gamma$ on the right-hand argument $x^{3}$, and the invariance of that argument under a subgroup. Additional proofs have appeared [6, 7].

In this article we show that (1.1) is one of six related hypergeometric identities, naturally indexed by an integer $N=2, \ldots, 7$. The $N$-th identity arises from the modular curve $X_{0}(N)$, in particular from its $N$-sheeted covering by $X_{0}\left(N^{2}\right)$. Here the curve $X_{0}(N)$, which classifies $N$-isogenies of elliptic curves over $\mathbb{C}$, is the (compactified) quotient of the upper half-plane $\mathfrak{H}$ by the Hecke congruence subgroup $\Gamma_{0}(N)$ of $\Gamma$. The connection to the Borweins' proof should be evident. Of the six identities, the identity (1.1) is associated with $X_{0}(3)$, and the ones associated with $X_{0}(2)$ and $X_{0}(4)$ were also found by Ramanujan. (They are the quadratic iteration in signature 4 and the quartic one in signature 2, respectively.) The identities

Received by the editors January 24, 2005 and, in revised form, July 18, 2005.

2000 Mathematics Subject Classification. Primary 11F03; 11F20, 33C05.

The author was supported in part by NSF Grant No. PHY-0099484.

(C) 2007 American Mathematical Society Reverts to public domain 28 years from publication 
associated with $X_{0}(N), N=5,6,7$, are new. The reason for their not having been discovered previously may be that they are most briefly expressed not in terms of ${ }_{2} F_{1}$, but rather in terms of $H l$, the so-called local Heun function [26]. The functions ${ }_{2} F_{1}, H l$ are solutions of canonical Fuchsian differential equations on $\mathbb{P}^{1}(\mathbb{C})$ with three and four singular points, respectively.

Each identity is a modular equation. When $N=2,3,4,5$, the curve $X_{0}\left(N^{2}\right)$ is of genus 0 and has a Hauptmodul (global uniformizing parameter), in terms of which the identity can be expressed. When $N=6,7$, the curve $X_{0}\left(N^{2}\right)$ is of genus 1 but its quotient $X_{0}^{+}\left(N^{2}\right)$ under the Fricke involution has a Hauptmodul. So the $N=6,7$ identities can be formulated without elliptic functions by including algebraic constraints. All of these hypergeometric identities can be viewed as algebraic transformations of series, but the following statement of the quintic $(N=5)$ identity in series language makes it clear how difficult it would be to prove them by series manipulation. The accompanying proof is really a verification and will be replaced in the sequel by a derivation based on Hauptmoduln. The function $h_{5}$ can be expressed in terms of $H l$ or ${ }_{2} F_{1}$. It will be shown that $h_{5}(z)=\left[\frac{1}{5}\left(z^{2}+10 z+5\right)\right]^{-1 / 4}{ }_{2} F_{1}\left(\frac{1}{12}, \frac{5}{12} ; 1 ; 1728 z /\left(z^{2}+10 z+5\right)^{3}\right)$.

Proposition 1.1. Let $h_{5}$, a $\mathbb{C}$-valued function, be defined in a neighborhood of $0 \in \mathbb{C}$ by $h_{5}(z)=\sum_{n=0}^{\infty} c_{n} z^{n}$, where the coefficients satisfy the three-term recurrence

$$
(2 n-1)^{2} c_{n-1}+2\left(44 n^{2}+22 n+5\right) c_{n}+500(n+1)^{2} c_{n+1}=0,
$$

initialized by $c_{-1}=0$ and $c_{0}=1$. Then for all $x$ in a neighborhood of 0 ,

$$
\begin{aligned}
& h_{5}\left(x\left(x^{4}+5 x^{3}+15 x^{2}+25 x+25\right)\right) \\
& =5\left[x^{4}+5 x^{3}+15 x^{2}+25 x+25\right]^{-1 / 2} h_{5}\left(\frac{x^{5}}{x^{4}+5 x^{3}+15 x^{2}+25 x+25}\right) .
\end{aligned}
$$

That is, $h_{5}$ satisfies a quintic functional equation.

Remark 1.1.1. The sequence $d_{n}:=500^{n} c_{n}, n \geq 0$, of Maclaurin coefficients of $h_{5}(500 z)$ is an integral sequence. It begins $1,-10,230,-6500,199750,-6366060$, $204990300,-6539387400, \ldots$.

Proof of Proposition 1.1. In a neighborhood of $z=0, h_{5}=h_{5}(z)$ is analytic and satisfies the second-order differential equation

$$
\left\{D_{z}^{2}+\left[\frac{1}{z}+\frac{z+11}{z^{2}+22 z+125}\right] D_{z}+\left[\frac{z+10}{4 z\left(z^{2}+22 z+125\right)}\right]\right\} h_{5}=0,
$$

as can be verified by termwise differentiation of its defining series. By changing variables in (1.4), it can be shown that as functions of $x$, the two sides of (1.3) satisfy a common differential equation, namely

$$
\left\{D_{x}^{2}+\left[\frac{1}{x}+\frac{D_{x} A(x)}{A(x)}+\frac{D_{x} B(x)}{2 B(x)}\right] D_{x}+\left[\frac{25(x A(x)+10)}{4 x A(x) B(x)}\right]\right\} f=0,
$$

with $A(x):=x^{4}+5 x^{3}+15 x^{2}+25 x+25$ and $B(x):=x^{2}+2 x+5$. The point $x=0$ is a regular singular point of (1.5), with characteristic exponents 0,0 . It follows from the theory of Fuchsian differential equations that in a neighborhood of $x=0$, there is a unique analytic solution of (1.5) that equals unity at $x=0$. But both sides of (1.3) are analytic at $x=0$ and equal unity there; so each can be identified with this unique solution, and they must equal each other. 
This article is organized as follows. Basic facts about modular curves and their coverings are summarized in Section 2. In Sections 3 and 4 the covering of $X_{0}(N)$ by $X_{0}\left(N^{2}\right)$ is reviewed, and useful lemmas are proved. Section 5 contains the key results, Theorems 5.1 and 5.3. which apply to $N=2,3,4,5$ and $N=6,7$ respectively. The algebraic hypergeometric transformations derived from these theorems are worked out in Sections 6.1 and 6.2. The derivation relies on a number of formulas relating canonical Hauptmoduln, which are collected in an appendix. In Section 7 some possible extensions are indicated.

\section{Curves And COverings}

The left action of $S L(2, \mathbb{Z})$ on $\left(\begin{array}{l}\tau_{1} \\ \tau_{2}\end{array}\right)$ with $\tau_{1}, \tau_{2} \in \mathbb{C}^{*}$ independent over $\mathbb{R}$, i.e., on elliptic curves of the form $\mathbb{C} /\left\langle\tau_{1}, \tau_{2}\right\rangle$, induces a projective action of the modular group $\Gamma:=S L(2, \mathbb{Z}) /\{ \pm I\}$ on the upper half-plane $\mathfrak{H} \ni \tau:=\tau_{1} / \tau_{2}$. The quotient $\Gamma \backslash \mathfrak{H}$ is the space of isomorphism classes of elliptic curves over $\mathbb{C}$, and its compactification $\Gamma \backslash\left(\mathfrak{H}^{*}:=\mathfrak{H} \cup \mathbb{Q} \cup\{\mathrm{i} \infty\}\right)$, with cusps, is the modular curve $X(1)$.

The isomorphism classes of $N$-isogenies of elliptic curves $\phi: E \rightarrow E^{\prime}$ with $N>1$, i.e., isogenies with kernel equal to the cyclic group $C_{N}$, are similarly classified by the non-cusp points of the modular curve $X_{0}(N):=\Gamma_{0}(N) \backslash \mathfrak{H}^{*}$, where $\Gamma_{0}(N)=$ $\left\{\left(\begin{array}{ll}a & b \\ c & d\end{array}\right) \in \Gamma|N| c\right\}$ is the $N$-th congruence subgroup. For any such $N$-isogeny of elliptic curves $\phi: E \rightarrow E^{\prime}$, i.e., $\phi: \mathbb{C} /\langle\tau, 1\rangle \rightarrow \mathbb{C} /\left\langle\tau^{\prime}, 1\right\rangle$, there is a dual one $\phi^{*}: \mathbb{C} /\left\langle\tau^{\prime}, 1\right\rangle \rightarrow \mathbb{C} /\langle\tau, 1\rangle$. This correspondence yields the Fricke involution $w_{N}$ on $X_{0}(N)$, defined as $E \leftrightarrow E^{\prime}$. On the level of the unquotiented half-plane $\mathfrak{H} \ni \tau$ it is simply the map $\tau \mapsto-1 / N \tau$. The curve $X_{0}^{+}(N)$ is defined as the quotient of $X_{0}(N)$ by $\left\langle w_{N}\right\rangle$, the group of two elements generated by $w_{N}$. Each non-cusp point of $X_{0}^{+}(N)$ corresponds to an unordered pair $\left\{\phi: E \rightarrow E^{\prime}, \phi^{*}: E^{\prime} \rightarrow E\right\}$.

The curve $X(1)$ is of genus zero, so its function field is singly generated; the generator can be taken to be the Klein-Weber $j$-invariant. This is effectively a meromorphic function on $\mathfrak{H}^{*}$ with an expansion about $\tau=\mathrm{i} \infty$ that begins $q^{-1}+\cdots$, where $q:=e^{2 \pi \mathrm{i} \tau}$ is the local uniformizing parameter. For any $N>1$, the function field of $X_{0}(N)$ is $\mathbb{C}\left(j, j_{N}\right)$, where $j_{N}(\tau):=j(N \tau)$. The Fricke involution $w_{N}$ interchanges $j, j_{N}$. If $g\left(X_{0}(N)\right)=0$, then $X_{0}(N)$ will have a Hauptmodul, which may be denoted $x_{N}=x_{N}(\tau)$, and both $j$ and $j_{N}$ will necessarily be rational functions of it. Being univalent, $x_{N}$ can be chosen to have a simple zero (resp. pole) at the cusp $\tau=\mathrm{i} \infty$ (resp. $\tau=0$ ). Since these two cusps are interchanged by $w_{N}, x_{N}$ can be defined so that $x_{N}(\tau) x_{N}(-1 / N \tau)=\kappa_{N}$ for any specified $\kappa_{N} \in \mathbb{Q}^{*}$.

If $N \mid N^{\prime}$, then $\Gamma_{0}(N) \geqslant \Gamma_{0}\left(N^{\prime}\right)$, yielding a (ramified) covering of $X_{0}(N)$ by $X_{0}\left(N^{\prime}\right)$ and an injection of the function field of $X_{0}(N)$ into that of $X_{0}\left(N^{\prime}\right)$. If, for example, both have Hauptmoduln, then $x_{N}$ will be a rational function of $x_{N^{\prime}}$. If $g\left(X_{0}\left(N^{\prime}\right)\right)>0$ but $g\left(X_{0}^{+}\left(N^{\prime}\right)\right)=0$, then $X_{0}\left(N^{\prime}\right)$ will be a hyperelliptic curve that doubly covers $X_{0}^{+}\left(N^{\prime}\right)$, and $X_{0}^{+}\left(N^{\prime}\right)$ will have a Hauptmodul $t_{N^{\prime}}$ such that $X_{0}\left(N^{\prime}\right)$ is defined by $s_{N^{\prime}}^{2}=\mathrm{P}\left(t_{N^{\prime}}\right)$ for some polynomial P of degree $2 g\left(X_{0}\left(N^{\prime}\right)\right)+2$. The hyperelliptic involution $s_{N^{\prime}} \mapsto-s_{N^{\prime}}$ will be the Fricke involution $w_{N^{\prime}}$. The function field of $X_{0}\left(N^{\prime}\right)$ will be generated by $t_{N^{\prime}}, s_{N^{\prime}}$; and like $s_{N^{\prime}}$, the Hauptmodul $x_{N}$ of $X_{0}(N)$ will be a two-valued algebraic function of $t_{N^{\prime}}$.

This article will focus on the especially interesting case $N^{\prime}=N^{2}$. When $N=2,3,4$, or 6 , the automorphism group $\Gamma_{0}\left(N^{2}\right)$ is conjugate in $\operatorname{PSL}(2, \mathbb{R})$ to the level- $N$ principal congruence subgroup $\Gamma(N)$ of $\Gamma$, so the corresponding curves 
$X_{0}\left(N^{2}\right), X(N)$ are isomorphic; and for any $p$, the quotient curve $X_{0}^{+}\left(p^{2}\right)$ is isomorphic to the arithmetically important curve $X_{\text {split }}(p)$. Computational treatment of the covering of $X_{0}(N)$ by $X_{0}\left(N^{2}\right)$ is facilitated by the many known formulas relating the associated Hauptmoduln, originating largely with Fricke. These do not appear in full in any modern reference, so they are reproduced here in an appendix, in an enhanced format. Each $N>1$ with $g\left(X_{0}(N)\right)=0$ and either $g\left(X_{0}\left(N^{2}\right)\right)=0$ or $g\left(X_{0}^{+}\left(N^{2}\right)\right)=0$ is included. The values of $N$ turn out to be $N=2, \ldots, 7$.

The genus $g\left(X_{0}(N)\right)$ here comes from the Hurwitz formula, or directly from Euler's theorem [27. The covering $j: X_{0}(N) \rightarrow \mathbb{P}^{1}(\mathbb{C}) \cong X(1)$ is $\psi(N)$-sheeted, where $\psi(N):=N \prod_{p \mid N}(1+1 / p)$ is the index $\left[\Gamma: \Gamma_{0}(N)\right]$. It is ramified only above the cusp $j=\infty$ and the elliptic fixed points $j=0,12^{3}$, corresponding to equianharmonic and lemniscatic elliptic curves respectively, i.e., only above the vertices $\tau=\mathrm{i} \infty, \rho:=e^{2 \pi i / 3}$, i of the fundamental half-domain of $\Gamma$ in $\mathfrak{H}^{*}$. The fibre above $j=\infty$ consists of $\sigma_{\infty}(N):=\sum_{d \mid N} \varphi((d, N / d))$ cusps, where $\varphi$ is the Euler totient function, i.e., $\varphi(N):=N \prod_{p \mid N}(1-1 / p)$. The fibre above $j=0$ (resp. $j=12^{3}$ ) includes $\varepsilon_{\rho}(N)$ cubic elliptic points (resp. $\varepsilon_{\mathrm{i}}(N)$ quadratic ones), each with unit multiplicity; other points, if any, have cubic (resp. quadratic) multiplicity. Here

$$
\varepsilon_{\rho}(N):=\left\{\begin{array}{ll}
\prod_{p \mid N}\left(1+\left(\frac{-3}{p}\right)\right), & 9 \nmid N, \\
0, & 9 \mid N,
\end{array} \quad \varepsilon_{\mathrm{i}}(N):= \begin{cases}\prod_{p \mid N}\left(1+\left(\frac{-1}{p}\right)\right), & 4 \nmid N, \\
0, & 4 \mid N,\end{cases}\right.
$$

with (:) the Legendre symbol. An application of Euler's theorem yields

$$
g\left(X_{0}(N)\right)=1+\frac{\psi(N)}{12}-\frac{\sigma_{\infty}(N)}{2}-\frac{\varepsilon_{\rho}(N)}{3}-\frac{\varepsilon_{\mathrm{i}}(N)}{4} .
$$

A further computation, first performed by Fricke [15, 20, reveals that in

$$
g\left(X_{0}^{+}(N)\right)=\frac{1}{2}\left[g\left(X_{0}(N)\right)+1\right]-\frac{1}{4} a(N),
$$

which follows from the Hurwitz formula, with $a(N)$ the number of fixed points of the Fricke involution on $X_{0}(N)$, the quantity $a(N)$ (when $N \geq 5$, at least) equals $h(-4 N)+h(-N)$ if $N \equiv-1(\bmod 4)$ and $h(-4 N)$ otherwise. Here $h(-d)$ is the class number of the imaginary quadratic field $\mathbb{Q}(\sqrt{-d})$.

\section{Liftings OF CUSPS}

The cusps of $X_{0}(N)$ have the following description [16, 24. The set of cusps of $\mathfrak{H}^{*}, \mathbb{P}^{1}(\mathbb{Q})=\mathbb{Q} \cup\{\mathrm{i} \infty\} \ni \tau$, is partitioned into classes, each equivalent under $\Gamma_{0}(N)$. A system of representatives, i.e., a choice of one from each class, may be taken to comprise certain fractions $\tau=\frac{a}{d}$ for each $d \mid N$, with $1 \leqslant a<d$ and $(a, d)=1$. Here $a$ is reduced modulo $f_{d, N}:=(d, N / d)$ while remaining coprime to $d$, so there are $\varphi((d, N / d))$ values of $a$, and hence $\varphi((d, N / d))$ cusps in $\mathbb{P}^{1}(\mathbb{Q})$ associated to $d$, which are inequivalent under $\Gamma_{0}(N)$. This is the source of the above formula for $\sigma_{\infty}(N)$. Each cusp of the form $\frac{a}{d}$ has width $e_{d, N}:=N / d f_{d, N}$, i.e., multiplicity $e_{d, N}$ above $X(1)$. That is, the fibre of the $\psi(N)$-sheeted cover $\pi_{N}: X_{0}(N) \rightarrow X(1)$ above the unique cusp of $X(1)$, located at $j=\infty$, includes (the equivalence class of) $\tau=\frac{a}{d}$ with multiplicity $e_{d, N}$. To emphasize that a cusp of $X_{0}(N)$ is an equivalence class, the notation $\left[\frac{a}{d}\right]$ will be used, or $\left[\frac{a}{d}\right]_{N}$ if the modular curve needs to be indicated. Since the 'distinguished' cusps $\tau=0$, i $\infty$ of $X_{0}(N)$ are equivalent to $\tau=\frac{1}{1}, \frac{1}{N}$ respectively, they may be written as $\left[\frac{1}{1}\right]_{N},\left[\frac{1}{N}\right]_{N}$. 
Now consider the inverse images of the $\sigma_{\infty}(N)$ cusps of $X_{0}(N)$ under its $N$ sheeted covering by $X_{0}\left(N^{2}\right)$, which will be denoted $\phi_{N}$. Note first that the covering $\pi_{N^{2}}: X_{0}\left(N^{2}\right) \rightarrow X(1)$, which has $\psi\left(N^{2}\right)=N \psi(N)$ sheets, satisfies $\pi_{N^{2}}=\pi_{N} \circ \phi_{N}$. The coverings $X_{0}\left(N^{2}\right) \stackrel{\phi_{N}}{\longrightarrow} X_{0}(N) \stackrel{\pi_{N}}{\longrightarrow} X(1)$ correspond to the subgroup relations $\Gamma_{0}\left(N^{2}\right)<\Gamma_{0}(N)<\Gamma$. For any $N, \Gamma_{0}(N)$ is normalized in $\operatorname{PSL}(2, \mathbb{R})$ by the Fricke involution $w_{N}: \tau \mapsto-1 / N \tau$. That is, if $\left(\begin{array}{ll}a & b \\ c & d\end{array}\right) \in \Gamma_{0}(N)$, then $\left(\begin{array}{cc}0 & -1 \\ N & 0\end{array}\right)^{-1}\left(\begin{array}{ll}a & b \\ c & d\end{array}\right)\left(\begin{array}{cc}0 & -1 \\ N & 0\end{array}\right) \in \Gamma_{0}(N)$, and $w_{N}$ induces a permutation of the cusps of $X_{0}(N)$. This interchanges any cusp $\left[\frac{a}{d}\right]$ with some cusp $\left[\frac{a^{\prime}}{d^{\prime}}\right]$, where $d^{\prime}=N / d$.

There is another covering by $X_{0}\left(N^{2}\right)$ that will be equally important. Though $\Gamma_{0}\left(N^{2}\right)$ is normalized by $w_{N^{2}}, \Gamma_{0}(N)$ is not: it is conjugated to an isomorphic subgroup $\Gamma_{0}(N)^{\prime}:=w_{N^{2}}^{-1} \Gamma_{0}(N) w_{N^{2}}$ of $\operatorname{PSL}(2, \mathbb{R})$. Since $\Gamma_{0}\left(N^{2}\right)<\Gamma_{0}(N)^{\prime}$, there is a corresponding cover $X_{0}\left(N^{2}\right) \stackrel{\phi_{N}^{\prime}}{\longrightarrow} X_{0}(N)^{\prime}$, where $X_{0}(N)^{\prime}:=\Gamma_{0}(N)^{\prime} \backslash \mathfrak{H}^{*}$. Since $\Gamma_{0}(N)^{\prime}$, unlike $\Gamma_{0}(N)$, is not a subgroup of the modular group $\Gamma$, the curve $X_{0}(N)^{\prime}$ does not naturally cover $X(1)$. But if $X_{0}(N)$ has a Hauptmodul $x_{N}$, which may be regarded as a $\Gamma_{0}(N)$-invariant function $x_{N}(\tau)$ on $\mathfrak{H}^{*}$, then $X_{0}(N)^{\prime}$ will too, and it may be chosen to be the $X_{0}(N)^{\prime}$-invariant function $x_{N}^{\prime}(\tau):=x_{N}(N \tau)$. This is because $\Gamma_{0}(N)^{\prime}=w^{-1} \Gamma_{0}(N) w$ where $w=\left(\begin{array}{cc}N & 0 \\ 0 & 1\end{array}\right)$, i.e., $w$ is the map $\tau \mapsto N \tau$.

The cusps of $X_{0}(N), X_{0}(N)^{\prime}$, which are subsets of $\mathbb{P}^{1}(\mathbb{Q})$ invariant under $\Gamma_{0}(N)$, $\Gamma_{0}(N)^{\prime}$, are related thus: $[\tau]_{N}$ is a cusp of $X_{0}(N)$ iff $[\tau]_{N} / N:=\left\{x / N \mid x \in[\tau]_{N}\right\}$ is a cusp of $X_{0}(N)^{\prime}$. The following lemma specifies how the two most important cusps of $X_{0}(N)$ (resp. $\left.X_{0}(N)^{\prime}\right)$ are lifted to formal sums of cusps of $X_{0}\left(N^{2}\right)$.

Lemma 3.1. Inverse images of the distinguished cusps $\tau=0, \mathrm{i} \infty$ on $X_{0}(N)$ (i.e., of $\left.\left[\frac{1}{1}\right]_{N},\left[\frac{1}{N}\right]_{N}\right)$ under the $N$-sheeted cover $\phi_{N}: X_{0}\left(N^{2}\right) \rightarrow X_{0}(N)$ are given by

$$
\phi_{N}{ }^{-1}\left(\left[\frac{1}{1}\right]_{N}\right)=N \cdot\left[\frac{1}{1}\right]_{N^{2}}, \quad \quad \phi_{N}^{-1}\left(\left[\frac{1}{N}\right]_{N}\right)=\sum_{\substack{d \text { s.t. } N|d| N^{2}, a=a_{1}, \ldots, a_{\varphi}\left(f_{d, N^{2}}\right)}}\left[\frac{a}{d}\right]_{N^{2}},
$$

where the right-hand sides list cusps of $X_{0}\left(N^{2}\right)$, and premultiplication by $N$ indicates multiplicity. The sum is over $(i) d$ such that $d \mid N^{2}$ with $N \mid d$, and (ii) the corresponding $\varphi\left(f_{d, N^{2}}\right)$ values of a, giving $N$ terms in all. Inverse images of the cusps $\tau=0$, i $\infty$ on $X_{0}(N)^{\prime}$ under $\phi_{N}^{\prime}: X_{0}\left(N^{2}\right) \rightarrow X_{0}(N)^{\prime}$ are similarly given by

$$
\phi_{N}^{\prime-1}\left(\left[\frac{1}{1}\right]_{N} / N\right)=\sum_{\substack{d \text { s.t. } d|N| N^{2}, a=a_{1}, \ldots, a_{\varphi}\left(f_{d, N^{2}}\right)}}\left[\frac{a}{d}\right]_{N^{2}}, \quad \phi_{N}^{\prime}{ }^{-1}\left(\left[\frac{1}{N}\right]_{N} / N\right)=N \cdot\left[\frac{1}{N^{2}}\right]_{N^{2}} \text {. }
$$

The sum is over $d$ such that $d \mid N$, and over the corresponding $\varphi\left(f_{d, N^{2}}\right)$ values of a.

Proof. For the duration of this proof, write any cusp $\left[\frac{a}{d}\right]$ as $\left(\begin{array}{l}a \\ d\end{array}\right) \in \mathbb{Z}^{2}$ to permit left-multiplication by elements of $S L(2, \mathbb{Z})$. The cosets of $\Gamma_{0}(N)$ in $\Gamma_{0}\left(N^{2}\right)$ are represented by $\left(\begin{array}{cc}1 & 0 \\ k N & 1\end{array}\right), k=0, \ldots, N-1$. The cusp $\left(\begin{array}{l}1 \\ 1\end{array}\right)$ of $X_{0}(N)$ is lifted by $\phi_{N}$ to the $N$ cusps $\mathfrak{c}_{k}:=\left(\begin{array}{cc}1 & 0 \\ k N & 1\end{array}\right)\left(\begin{array}{l}1 \\ 1\end{array}\right)=\left(\begin{array}{c}1 \\ k N+1\end{array}\right), k=0, \ldots, N-1$. To prove they are the same in $X_{0}\left(N^{2}\right)$, it suffices to find $\gamma_{k} \in \Gamma_{0}\left(N^{2}\right)$ such that $\gamma_{k} \cdot \mathfrak{c}_{k}=\mathfrak{c}_{0}$. By examination, $\left(\begin{array}{cc}k^{2} N^{2}+k N+1 & -k N \\ k^{2} N^{2} & -k N+1\end{array}\right)$ will work.

The lifting of $\left(\begin{array}{c}1 \\ N\end{array}\right)$ similarly comprises the $N$ cusps $\mathfrak{d}_{k}:=\left(\begin{array}{cc}1 & 0 \\ k N & 1\end{array}\right)\left(\begin{array}{l}1 \\ N\end{array}\right)=\left(\begin{array}{c}1 \\ (k+1) N\end{array}\right)$, $k=0, \ldots, N-1$, of $X_{0}\left(N^{2}\right)$. Since $w_{N^{2}} \mathfrak{d}_{k}=\left(\begin{array}{c}-(k+1) N \\ N^{2}\end{array}\right) \sim\left(\begin{array}{c}-(k+1) \\ N\end{array}\right)$, the cusps $\left\{w_{N^{2}} \mathfrak{d}_{k}\right\}_{k=0}^{N-1}$ are of the form $\left(\begin{array}{l}a \\ d\end{array}\right)$, where $d$ runs over the divisors of $N$ and $a$ runs over 
the $\varphi(d)=\varphi\left(\left(d, N^{2} / d\right)\right)$ integers in the range $1, \ldots, d-1$ that are relatively prime to $d$. As noted, $w_{N^{2}}$ maps any cusp $\left(\begin{array}{l}a \\ d\end{array}\right)$ of $\Gamma_{0}\left(N^{2}\right)$ to a cusp $\left(\begin{array}{c}a^{\prime} \\ d^{\prime}\end{array}\right)$ with $d^{\prime}=N^{2} / d$; so applying $w_{N^{2}}$ again yields the given formula for the formal sum $\sum_{k=0}^{N-1} \mathfrak{d}_{k}$.

The statements concerning liftings by $\phi_{N}^{\prime}$ can be proved likewise, or simply by applying $w_{N^{2}}$ to both sides of each of the two previously derived formulas.

The following lemma subsumes the half of Lemma 3.1 that deals with $\phi_{N}$. It can be proved by a similar argument.

Lemma 3.2. The fibre of $\phi_{N}$ over any cusp of $X_{0}(N)$ of the form $\left[\frac{a}{d}\right]_{N}$ with $d \mid N$ consists of $d /(d, N / d)$ cusps of $X_{0}\left(N^{2}\right)$, each of the form $\left[\frac{\tilde{a}}{d}\right]_{N^{2}}$ with $\tilde{d} \mid N^{2}$, where $\tilde{d}$ is constrained to satisfy $d=(\tilde{d}, N)$. Each of these cusps of $X_{0}\left(N^{2}\right)$ appears in the fibre with multiplicity equal to the width quotient $e_{d, N^{2}} / e_{d, N}=N(d, N / d) / d$.

A consequence of the lemma is that for any $d \mid N$, the inverse image under $\phi_{N}$ of the set consisting of the $\varphi((d, N / d))$ cusps of $X_{0}(N)$ of the form $\left[\frac{a}{d}\right]_{N}$ is a set consisting of the $[d /(d, N / d)] \varphi((d, N / d))$ cusps of $X_{0}\left(N^{2}\right)$ of the form $\left[\frac{\tilde{a}}{\tilde{d}}\right]_{N^{2}}$, where $\tilde{d}$ ranges over the solutions of $d=(\tilde{d}, N)$ with $\tilde{d} \mid N^{2}$. Each of these cusps of $X_{0}\left(N^{2}\right)$ appears in the inverse image with multiplicity $N(d, N / d) / d$.

\section{Liftings OF DiffERENTIAL OPERATORS}

4.1. Some background. The coordinate $\tau$ on $\mathfrak{H}^{*}$ can be viewed as a multivalued function on any algebraic curve $X_{1}$ of the form $\Gamma_{1} \backslash \mathfrak{H}^{*}$, where $\Gamma_{1}$ is a Fuchsian subgroup (of the first kind) of the automorphism group $P S L(2, \mathbb{R})$. It is well known that on $X_{1}, \tau$ will locally equal the ratio of two independent solutions of an appropriate second-order Fuchsian differential equation [14, 19. For example, consider the case $\Gamma_{1}=\Gamma$, since $X_{1}=X(1)$ is parametrized by the $j$-invariant and the equation may be written in terms of the derivation $D_{j}:=d / d j$. Actually, it is more convenient to use the Hauptmodul $\hat{J}:=12^{3} / j$, the reciprocal of Klein's invariant $J=j / 12^{3}$.

Proposition 4.1. In a neighborhood of any point on $X(1)=\Gamma \backslash \mathfrak{H}^{*} \ni \hat{J}$, any branch of $\tau$ equals the ratio $u_{1} / u_{2}$ of two independent local solutions of

$$
L_{\frac{1}{12}, \frac{5}{12} ; 1} u:=\left\{D_{\hat{J}}^{2}+\left[\frac{1}{\hat{J}}+\frac{1}{2(\hat{J}-1)}\right] D_{\hat{J}}+\frac{5 / 144}{\hat{J}(\hat{J}-1)}\right\} u=0 .
$$

Moreover, any such ratio is of the form $(a \tau+b) /(c \tau+d)$ with $a d \neq b c$.

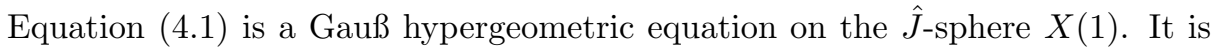
Fuchsian, with regular singular points at $\hat{J}=0,1, \infty$ and respective characteristic exponents 0,$0 ; 0, \frac{1}{2} ; \frac{1}{12}, \frac{5}{12}$. It will be referred to as ${ }_{2} \mathcal{E}_{1}\left(\frac{1}{12}, \frac{5}{12} ; 1\right)$, since the unique local solution analytic at $\hat{J}=0$ and normalized to unity there is the hypergeometric function ${ }_{2} F_{1}\left(\frac{1}{12}, \frac{5}{12} ; 1 ; \cdot\right)$. On $\mathfrak{H}^{*} \ni \tau$, the multivalued function ${ }_{2} F_{1}\left(\frac{1}{12}, \frac{5}{12} ; 1 ; \hat{J}(\tau)\right)$ can be shown to be proportional to $\hat{J}^{-1 / 2}(1-\hat{J})^{-1 / 4}(d \hat{J} / d \tau)^{1 / 2}$, and to be a weight1 modular form for $\Gamma$. The fractional powers are consistent with being multivalued.

The entire two-dimensional space of solutions of (4.1), viewed as functions of $\tau$, is ${ }_{2} F_{1}\left(\frac{1}{12}, \frac{5}{12} ; 1 ; \hat{J}(\tau)\right)(\mathbb{C} \tau+\mathbb{C})\left[32\right.$. By comparison, the space of solutions of $D_{\tau}^{2} \tilde{u}=0$ 
on the $\tau$-plane is $\mathbb{C} \tau+\mathbb{C}$. Lifting $L_{\frac{1}{12}, \frac{5}{12} ; 1} u=0$ from $X(1)$ to $\mathfrak{H}$ along the infinitesheeted covering map $\tau \mapsto \hat{J}$ will yield $D_{\tau}^{2} \tilde{u}=0$ only if the lifting is 'weak', i.e., if the dependent variable is altered according to $\tilde{u}={ }_{2} F_{1}\left(\frac{1}{12}, \frac{5}{12} ; 1 ; \hat{J}(\tau)\right)^{-1} \cdot u$.

Proposition 4.1 can be generalized from $X(1)=\Gamma \backslash \mathfrak{H}^{*}$ to any $X_{1}=\Gamma_{1} \backslash \mathfrak{H}^{*}$. There are two cases to be distinguished: when this algebraic curve is of genus zero, with function field $\mathbb{K}\left(X_{1}\right)=\mathbb{C}(x)$, where $x$ is any Hauptmodul, and when it is of positive genus, with function field $\mathbb{K}\left(X_{1}\right)=\mathbb{C}(x, y)$, where $x, y$ are related by some polynomial equation $\Phi(x, y)=0$ over $\mathbb{C}$.

Before stating the generalization, we recall the definition of characteristic exponents of a second-order differential operator $L=D_{x}^{2}+\mathcal{A} \cdot D_{x}+\mathcal{B}$ on $X_{1}$, where $\mathcal{A}, \mathcal{B} \in \mathbb{K}\left(X_{1}\right)$. Such an operator is said to be Fuchsian if all its singular points are regular, i.e., if it has two characteristic exponents $\alpha_{i, 1}, \alpha_{i, 2} \in \mathbb{C}$ (which may be the same) at each singular point $\mathfrak{s}_{i} \in X_{1}$. If $\alpha_{i, 1}-\alpha_{i, 2} \notin \mathbb{Z}$, this means $L u=0$ has local solutions $u_{i, j}, j=1,2$, at $\mathfrak{s}_{i}$ of the form $t^{\alpha_{i, j}}$ times an invertible function of $t$, where $t$ is a local uniformizing parameter (if $\alpha_{i, 1}-\alpha_{i, 2} \in \mathbb{Z}$, one solution may be logarithmic). For example, at each singular point with a zero exponent there is an analytic local solution, unique up to normalization. Each ordinary (i.e., non-singular) point has exponents 0,1 .

Theorem 4.2. In a neighborhood of any point on $X_{1}=\Gamma_{1} \backslash \mathfrak{H}^{*}$, any branch of the function $\tau$ equals the ratio $u_{1} / u_{2}$ of two independent local solutions of a second-order Fuchsian differential equation $L u:=\left(D_{x}^{2}+\mathcal{A} \cdot D_{x}+\mathcal{B}\right) u=0$, where $\mathcal{A}, \mathcal{B} \in \mathbb{K}\left(X_{1}\right)$. Moreover, any such ratio is of the form $(a \tau+b) /(c \tau+d)$ with $a d \neq b c$. One can choose $L$ so that its singular points are the fixed points of $\Gamma_{1}$, with the difference of characteristic exponents equaling $1 / k$ at each fixed point of order $k$, and zero at each parabolic fixed point (i.e., cusp).

This is a special case of a theorem dealing with Fuchsian automorphic functions of the first kind [14, $\S 44$, Thm. 15]. The coefficients $\mathcal{A}, \mathcal{B}$ of $L$ can be taken to be $0, \frac{1}{2}\{\tau, x\}$, where $\{\cdot, \cdot\}$ is the Schwarzian derivative. In this case the space of solutions of $L u=0$, regarded as functions of $\tau$, is $(d x / d \tau)^{1 / 2}(\mathbb{C} \tau+\mathbb{C})$. However, $L$ is not unique. Any substitution of the type $u \mapsto f^{\alpha} u$, where $f \in \mathbb{K}\left(X_{1}\right)$ and $\alpha \in \mathbb{C}$, will produce an operator with transformed coefficients $\hat{\mathcal{A}}, \hat{\mathcal{B}} \in \mathbb{K}\left(X_{1}\right)$, but the same solution ratios. Similarly, the Liouville substitution $u \mapsto u \exp \left[-\frac{1}{2} \int_{x_{0}}^{x} \mathcal{A} d x\right]$ will transform $\mathcal{A}, \mathcal{B}$, where $\mathcal{A}$ may be non-zero, to $0, \mathcal{B}-\frac{1}{2} D_{x} \mathcal{A}-\frac{1}{4} \mathcal{A}^{2}$. These substitutions will leave exponent differences invariant, though they may shift exponents.

Theorem 4.3. Let $L_{i}=D_{x}^{2}+\mathcal{A}_{i} \cdot D_{x}+\mathcal{B}_{i}, i=1,2$, where $\mathcal{A}_{i}, \mathcal{B}_{i} \in \mathbb{K}\left(X_{1}\right)$, be Fuchsian operators on $X_{1}=\Gamma_{1} \backslash \mathfrak{H}^{*}$ with the property that any ratio of independent solutions of $L_{1} u=0$ or of $L_{2} u=0$ is of the form $(a \tau+b) /(c \tau+d)$. Suppose that the singular points of each are the fixed points of $\Gamma_{1}$, with the difference of exponents equaling $1 / k$ at each elliptic fixed point of order $k$ and zero at each cusp. (i) If $\mathcal{A}_{1}=\mathcal{A}_{2}=0$, then $L_{1}, L_{2}$ are equal. (ii) If $X_{1}$ is of genus zero and $L_{1}, L_{2}$ have the same exponents (not merely exponent differences), then they are equal.

Part (i) is a special case of a theorem dealing with Fuchsian automorphic functions [14, $\S 111$, Thm. 7; and $\S 115]$. The uniqueness of $L$ is deduced from the requirement that at each point, the inverse of any solution ratio $x \mapsto u_{1} / u_{2}$, such as $x$ as a function of $\tau \in \mathfrak{H}$, must be single-valued. Part (ii) is proved by applying the above Liouville substitution to $L_{1}, L_{2}$, and then invoking part (i). 
To place this theorem in context, recall that any Fuchsian operator $L$ on $X_{1}$ of the form $D_{x}^{2}+\mathcal{A} \cdot D_{x}+\mathcal{B}$ determines a flat (i.e., integrable) holomorphic connection on a trivial 2-plane bundle over the punctured curve $X_{1}^{0}:=X_{1} \backslash\left\{\mathfrak{s}_{1}, \ldots, \mathfrak{s}_{n}\right\}$, where $\mathfrak{s}_{1}, \ldots, \mathfrak{s}_{n}$ are the singular points of $L$. The connection comes from analytically continuing any two independent local solutions $u_{1}, u_{2}$ along paths in $X_{1}^{0}$, producing an element of $G L(2, \mathbb{C})$ for each path. One may optionally quotient out the center $\mathbb{C}^{\times}$of $G L(2, \mathbb{C})$ to obtain a projective action: an element of the Möbius group $P G L(2, \mathbb{C})$, acting on the ratio $u_{1} / u_{2} \in \mathbb{P}^{1}(\mathbb{C})$. Such actions constitute a flat holomorphic connection on a trivial $\mathbb{P}^{1}(\mathbb{C})$-bundle over $X_{1}^{0}$.

In general, $L$ and the consequent flat connection on the trivial 2-plane bundle over $X_{1}^{0}$ are not uniquely determined by the $2 n-1$ independent exponents. In classical language, $L$ and the flat connection are parametrized by the exponents together with certain (complex) accessory parameters. The projectivized flat connection on the trivial $\mathbb{P}^{1}(\mathbb{C})$-bundle over $X_{1}^{0}$ will be parametrized by the $n$ exponent differences, together with certain of the accessory parameters. If $n \geq 3$ and $X_{1}$ is of genus $g$, the projectivized connection will depend on $n-3+3 g$ 'projective' accessory parameters. If the innocuous normalization $\mathcal{A}=0$ is imposed, then the remaining coefficient $\mathcal{B}$ will be naturally parametrized by the exponent differences and these accessory parameters. Part (i) of Theorem 4.3 is really a statement that if $x \mapsto u_{1} / u_{2}$ is to be the inverse of a single-valued function at each point, then the projective accessory parameters, and hence the flat connection on the trivial $\mathbb{P}^{1}(\mathbb{C})$-bundle, are uniquely determined by the exponent differences.

In the absence of any imposed normalization, the pair $\mathcal{A}, \mathcal{B}$, and hence $L$ itself and the flat connection on the trivial 2-plane bundle, will be parametrized by the $2 n-1$ independent exponents, the $n-3+3 g$ projective accessory parameters, and $g$ additional 'affine' accessory parameters. The presence of these parameters when $g>0$ is the reason for the restriction to $g=0$ in part (ii) of Theorem 4.3. Their values could differ between $L_{1}$ and $L_{2}$, even if those two differential operators have the same exponents and projective accessory parameters.

The following is an explanation of how the accessory parameters, both projective and affine, appear in any Fuchsian operator $L$ on $X_{1}$ of the form $D_{x}^{2}+\mathcal{A} \cdot D_{x}+\mathcal{B}$. If $X_{1}$ is of genus zero, suppose one of the $n \geq 3$ singular points is $x=\infty$ and that each finite singular point has one exponent equal to zero. (E.g., the operator $L_{\frac{1}{12}, \frac{5}{12} ; 1}$ of ${ }_{2} \mathcal{E}_{1}\left(\frac{1}{12}, \frac{5}{12} ; 1\right)$ has these properties.) Then $L$ will have the normal form [25]

$$
\frac{d^{2}}{d x^{2}}+\left[\sum_{i=1}^{n-1} \frac{1-\rho_{i}}{x-a_{i}}\right] \cdot \frac{d}{d x}+\left[\frac{\Pi_{n-3}(x)}{\prod_{i=1}^{n-1}\left(x-a_{i}\right)}\right] .
$$

Here $\left\{a_{i}\right\}_{i=1}^{n-1}$ are the finite singular points, with exponents $0, \rho_{i}$, and $\Pi_{n-3}(x)$ is a degree- $(n-3)$ polynomial. Its leading coefficient determines the exponents at $x=\infty$, and its $n-3$ trailing coefficients are the (projective) accessory parameters. If, on the other hand, $X_{1}$ is of positive genus, then suppose for simplicity it is elliptic $(g=1)$ or hyperelliptic $(g>1)$, with $\Phi(x, y)=y^{2}-\mathrm{P}_{2 g+2}(x)$, where $\mathrm{P}_{2 g+2}(x)$ is some polynomial of degree $2 g+2$ with simple roots. Then the generalization of (4.2) is straightforward. If the $n$ singular points include the infinite points $(x, y)=$ $(\infty, \pm \infty)$ and the $n-2$ finite points $(x, y)=\left(x_{i}, y_{i}\right)$, the exponents of the latter 
being $0, \rho_{i}$, then $L$ will necessarily be proportional to

$$
\begin{aligned}
\left(y \frac{d}{d x}\right)^{2}+\left[\sum_{i=0}^{n-2}\left(1-\rho_{i}\right)\right. & \left.\left(\frac{1}{2} \cdot \frac{y+y_{i}}{x-x_{i}}\right)+\Pi_{g}(x)\right] \cdot\left(y \frac{d}{d x}\right) \\
+ & {\left[\sum_{i=1}^{n-2} \mathfrak{b}_{i}\left(\frac{1}{2} \cdot \frac{y+y_{i}}{x-x_{i}}\right)+\Pi_{2 g}(x)+y \Pi_{g-1}(x)\right], }
\end{aligned}
$$

where the operator $y d / d x$ is the fundamental derivation on $\mathbb{C}(x, y)$, and the function $(1 / 2)\left(y+y_{i}\right) /\left(x-x_{i}\right)$ has simple poles at $\left(x_{i}, y_{i}\right)$ and $(\infty, \pm \infty)$. The three polynomials $\Pi_{g}(x), \Pi_{2 g}(x), \Pi_{g-1}(x)$ have respective degrees $g, 2 g, g-1$. Their leading coefficients determine the exponents of the two infinite points. The $g$ trailing coefficients of $\Pi_{g}(x)$ are the affine accessory parameters, whereas the $n-2$ coefficients $\left\{\mathfrak{b}_{i}\right\}_{i=1}^{n-2}$, the $2 g$ trailing coefficients of $\Pi_{2 g}(x)$, and the $g-1$ trailing coefficients of $\Pi_{g-1}(x)$, together make up the $n-3+3 g$ projective accessory parameters.

4.2. Weak liftings. We now specialize to the case when the Fuchsian group $\Gamma_{1}$ is a subgroup of the modular group $\Gamma$ and, in particular, to the case $\Gamma_{1}=\Gamma_{0}(N)$. By Theorem 4.2, it is possible to represent the coordinate $\tau$ of $\mathfrak{H}$ as a ratio of two solutions of some Fuchsian differential equation on $X_{0}(N)=\Gamma_{0}(N) \backslash \mathfrak{H}^{*}$. A differential equation with this property can be derived as the lifting, or any weak lifting, of ${ }_{2} \mathcal{E}_{1}\left(\frac{1}{12}, \frac{5}{12} ; 1\right)$ from $X(1)$ to $X_{0}(N)$. Under some circumstances, as will be explained in the next section, it is possible to derive a similar equation on $X_{0}\left(N^{2}\right)$ by either of two further liftings, the equivalence between which will yield a hypergeometric identity. Any two such equations are identical only if their exponents are the same; so we now consider the effects of liftings on exponents.

Suppose $L=D_{x}^{2}+\mathcal{A} \cdot D_{x}+\mathcal{B}$ is a differential operator on any algebraic curve $X_{1}=\Gamma_{1} \backslash \mathfrak{H}^{*}$ over $\mathbb{C}$ with derivation $D_{x}$ and function field $\mathbb{K} / \mathbb{C}$. Let $\xi: \tilde{X}_{1} \rightarrow X_{1}$ be the rational map of curves arising from a subgroup relation $\tilde{\Gamma}_{1} \leq \Gamma_{1}$. Here $\tilde{X}_{1}$ will have its own function field $\tilde{\mathbb{K}} / \mathbb{C}$ with derivation $D_{\tilde{x}}$. The lifting of $L$ to $\tilde{X}_{1}$ will be an operator $\tilde{L}=D_{\tilde{x}}^{2}+\tilde{\mathcal{A}} \cdot D_{\tilde{x}}+\tilde{\mathcal{B}}$ with $\tilde{\mathcal{A}}, \tilde{\mathcal{B}} \in \tilde{\mathbb{K}}$, satisfying the condition that $L u=0, \tilde{L} \tilde{u}=0$ locally have independent solution pairs $u_{1}, u_{2}$ and $\tilde{u}_{1}, \tilde{u}_{2}$ such that $\tilde{u}_{i}=u_{i} \circ \xi$. Informally, $\tilde{L}$ is obtained from $L$ by performing a change of (independent) variable, and also left-multiplying by an element of $\tilde{\mathbb{K}}^{\times}$if needed to preserve monicity. Each local solution $\tilde{u}(\cdot)$ of $\tilde{L} \tilde{u}=0$ on $\tilde{X}_{1}$ will be of the form $u(\xi(\cdot))$, where $u$ is some local solution of $L u=0$.

A differential operator $\tilde{M}$ of the same form as $\tilde{L}$ is said to be a weak lifting of $L$ if there are ratios $\sigma, \tilde{\sigma}$ of independent solutions of $L u=0, \tilde{M} \tilde{u}=0$ respectively, such that $\tilde{\sigma}=\sigma \circ \xi$. In a projective context, weak liftings are clearly the more appropriate concept. Informally, a weak lifting of the differential equation $L u=0$ to $\tilde{X}_{1}$ may incorporate a linear change of the dependent variable. For example, it may be multiplied by any $\tilde{f} \in \tilde{\mathbb{K}}^{\times}$. In this case any local solution $\tilde{u}(\cdot)$ of $\tilde{M} \tilde{u}=0$ on $\tilde{X}_{1}$ will be of the form $\tilde{f}(\cdot) u(\xi(\cdot))$, where $u$ is a local solution of $L u=0$.

From this point, we shall consider only lifting prefactors that are elements of $\tilde{\mathbb{K}}_{e}^{\times} \supset \tilde{\mathbb{K}}^{\times}$, the collection of (algebraic, multivalued) functions on $\tilde{X}_{1}$ of the form $\tilde{f}^{\alpha}$ with $\tilde{f} \in \tilde{\mathbb{K}}^{\times}$and $\alpha \in \mathbb{Q}$. The 'extension' $\tilde{\mathbb{K}}_{e}^{\times}$is not a field, due to the absence of closure under addition. Up to scalar multiplication, any $\tilde{f} \in \tilde{\mathbb{K}}^{\times}$may be identified with its divisor $\sum_{i} n_{i} \tilde{\mathfrak{p}}_{i}$, an element of the free $\mathbb{Z}$-module on $\tilde{X}_{1}$. Consequently, any $\tilde{f}_{e} \in \tilde{\mathbb{K}}_{e}^{\times}$has an associated 'generalized divisor' of the form $\sum_{i} c_{i} \tilde{\mathfrak{p}}_{i}$, an element 
of the free $\mathbb{Q}$-module on $\tilde{X}_{1}$. Since $\left(\tilde{f}^{\alpha}\right) \circ D_{\tilde{x}} \circ\left(\tilde{f}^{\alpha}\right)^{-1}=D_{\tilde{x}}-\alpha\left(D_{\tilde{x}} \tilde{f}\right) / \tilde{f}$, multiplying the dependent variable by any $\tilde{f}_{e} \in \tilde{\mathbb{K}}_{e}^{\times}$will yield a weak lifting $\tilde{M}$ that has the same general form as $\tilde{L}$, with coefficient functions $\tilde{\mathcal{A}}, \tilde{\mathcal{B}}$ that are elements of $\tilde{\mathbb{K}}$.

Lifting a Fuchsian operator $L=D_{x}^{2}+\mathcal{A} \cdot D_{x}+\mathcal{B}$ on $X_{1}$ to $\tilde{X}_{1}$ transforms exponents in a straightforward way. Suppose $\tilde{\mathfrak{p}} \in \tilde{X}_{1}$ is a critical point of $\xi: \tilde{X}_{1} \rightarrow X_{1}$ with corresponding critical value $\mathfrak{p} \in X_{1}$, i.e., suppose $\xi(\tilde{t})$ equals $t^{n}$ times an invertible function of $t$, where $\tilde{t}, t$ are local uniformizing parameters near $\tilde{\mathfrak{p}}, \mathfrak{p}$ respectively, and $n>1$ is the ramification index. Then the exponents of the lifting $\tilde{L}$ at $\tilde{\mathfrak{p}} \in \tilde{X}_{1}$ will be $n$ times those of $L$ at $\mathfrak{p} \in X_{1}$. (This statement extends to the case when $\tilde{\mathfrak{p}}$ is not a critical point of $\xi$ and $n=1$.) If the lifting prefactor $\tilde{f}_{e} \in \tilde{\mathbb{K}}_{e}^{\times}$has generalized divisor $\sum_{i} c_{i} \tilde{\mathfrak{p}}_{i}$, where $c_{i} \in \mathbb{Q}$ and $\tilde{\mathfrak{p}}_{i} \in \tilde{X}_{1}$, then the exponents of the weak lifting $\tilde{M}$ at each point $\tilde{\mathfrak{p}}_{i}$ will be shifted by $c_{i}$, relative to those of the lifting $\tilde{L}$.

Most previous work on Fuchsian differential equations on modular curves has adhered to a convention taken from conformal mapping, according to which the equation should be of the form $\left(D_{x}^{2}+\mathcal{B}\right) u=0$, i.e., should be formally selfadjoint, with $\mathcal{A}=0$ [19]. This typically forces each exponent to be non-zero. To derive hypergeometric identities, it is better to adopt an asymmetric convention informally introduced in (4.2)-(4.3): every singular point but one should have a zero exponent.

Definition 4.4. A Fuchsian operator $L=D_{x}^{2}+\mathcal{A} \cdot D_{x}+\mathcal{B}$ on an algebraic curve $X_{1}$ over $\mathbb{C}$ is said to be in normal form relative to a specified point $\underline{\infty} \in X_{1}$ (typically, a singular point) if each singular point of $L$ not equal to $\underline{\infty}$ has a zero exponent.

Requiring a weak lifting to be in normal form comes close to specifying it uniquely. This will not be explored further here, since for the purpose of studying weak liftings from one modular curve to another, the following lemma will suffice.

Lemma 4.5. Suppose a Fuchsian operator $L=D_{x}^{2}+\mathcal{A} \cdot D_{x}+\mathcal{B}$ on $X_{1}$ is in normal form relative to a specified point $\underline{\infty} \in X_{1}$, with $\alpha_{\infty}$ denoting one of the exponents of $L$ at $\underline{\infty}$. Let $\xi: \tilde{X}_{1} \rightarrow X_{1}$ be a rational map of algebraic curves over $\mathbb{C}$. If $\tilde{f} \in \tilde{\mathbb{K}}$ is a function with divisor equal to $\sum_{\tilde{\mathfrak{p}} \in \xi^{-1}(\underline{\infty})}[(\tilde{\mathfrak{p}})-(\underline{\widetilde{\alpha}})]$, for some $\underline{\widetilde{\infty}} \in \xi^{-1}(\underline{\infty})$, then the weak lifting $\tilde{M}=D_{\tilde{x}}^{2}+\tilde{\mathcal{A}} \cdot D_{\tilde{x}}+\tilde{\mathcal{B}}$ of $L$ to $\tilde{X}_{1}$ induced by $\tilde{u}(\cdot)=\tilde{f}(\cdot)^{-\alpha_{\infty}} u(\xi(\cdot))$ will be in normal form relative to $\underline{\widetilde{\Phi}}$.

Moreover, for any singular point $\tilde{O} \notin \xi^{-1}(\underline{\infty})$ on $\tilde{X}_{1}$, the unique local solution $\tilde{u}_{0}$ of $\tilde{M} \tilde{u}=0$ which is analytic and equal to unity at $\tilde{O}$ equals $\tilde{f}(\cdot)^{-\alpha_{\infty}} u_{0}(\xi(\cdot))$, where $u_{0}$ is the corresponding analytic local solution of $L u=0$ at $O:=\xi(\tilde{O})$.

Proof. The first statement follows from a straightforward computation of the lifted exponents, and the second from the preservation of analyticity of local solutions at each singular point other than $\underline{\infty}$, under lifting.

Lemma 4.6. Let $\xi: \mathbb{P}^{1}(\mathbb{C})_{\tilde{x}} \rightarrow \mathbb{P}^{1}(\mathbb{C})_{x}$ be a rational map from the $\tilde{x}$-sphere to the $x$-sphere, and suppose the operator $L=D_{x}^{2}+\mathcal{A}(x) D_{x}+\mathcal{B}(x)$ on the $x$-sphere is in normal form relative to the point $x=\infty$. Let $\alpha_{\infty}$ denote one of the two exponents of $L u=0$ at $x=\infty$. If $\xi(\tilde{x})=P(\tilde{x}) / Q(\tilde{x})$ with $P, Q$ having no nontrivial factor in common, then the weak lifting $\tilde{M}$ of $L$ to the $\tilde{x}$-sphere induced by $\tilde{u}(\cdot)=$ $Q(\cdot)^{-\alpha_{\infty}} u(\xi(\cdot))$ will be in normal form relative to the point $\tilde{x}=\infty$.

Moreover, for any singular point $\tilde{x}=\tilde{O}$ with $\xi(\tilde{O}) \neq \infty$, the unique local solution $\tilde{u}_{0}$ of $\tilde{M} \tilde{u}=0$ analytic and equal to unity at $\tilde{x}=\tilde{O}$ equals $Q(\cdot)^{-\alpha_{\infty}} u_{0}(\xi(\cdot))$, where $u_{0}$ is the corresponding analytic local solution of $L u=0$ at $O:=\xi(\tilde{O})$. 
Proof. This is a specialization of Lemma 4.5 to the case of zero genus.

As the appendix summarizes, if $g\left(X_{0}(N)\right)=0$, then $X_{0}(N)$ is coordinatized by a Hauptmodul $x_{N}$ with divisor (i $\left.\infty\right)$ - (0), i.e., a univalent function $x_{N}$ with a simple zero (resp. pole) at the cusp $\tau=\mathrm{i} \infty$ (resp. the cusp $\tau=0$ ). The covering map $\pi_{N}: X_{0}(N) \rightarrow X(1)$ is given by a rational function $j=j\left(x_{N}\right)=P_{N}\left(x_{N}\right) / Q_{N}\left(x_{N}\right)$, a quotient of monic polynomials satisfying $\operatorname{deg} P_{N}=\psi(N), \operatorname{deg} P_{N}-\operatorname{deg} Q_{N}=N$, and $x_{N} \mid Q_{N}\left(x_{N}\right)$. So $\hat{J}=\hat{J}\left(x_{N}\right)=1728 Q_{N}\left(x_{N}\right) / P_{N}\left(x_{N}\right)$. The cusps on the $x_{N}$-sphere $X_{0}(N)$ comprise the zeroes of $Q_{N}$, including $x_{N}=0$, and also $x_{N}=\infty$.

Definition 4.7. If $X_{0}(N)$ is of genus zero, the fundamental analytic function $h_{N}$, which will play a major role in the sequel, is defined by

$$
h_{N}\left(x_{N}\right):=\left[P_{N}\left(x_{N}\right) / P_{N}(0)\right]^{-1 / 12}{ }_{2} F_{1}\left(\frac{1}{12}, \frac{5}{12} ; 1 ; \frac{1728 Q_{N}\left(x_{N}\right)}{P_{N}\left(x_{N}\right)}\right)
$$

in a neighborhood of the distinguished cusp $x_{N}=0$ of $X_{0}(N)$, i.e., of $\tau=\mathrm{i} \infty$.

Proposition 4.8. If $X_{0}(N)$ is of genus zero, there is a normal-form weak lifting $\tilde{M}_{N} \tilde{u}=0$ of ${ }_{2} \mathcal{E}_{1}\left(\frac{1}{12}, \frac{5}{12} ; 1\right)$ from the $\hat{J}$-sphere $X(1)$ to $X_{0}(N)$, along $\pi_{N}$, which has a total of $\left(\sigma_{\infty}+\varepsilon_{\mathrm{i}}+\varepsilon_{\rho}\right)(N)$ singular points on $X_{0}(N)$, classified thus:

(1) one singular point with characteristic exponents $\frac{1}{12} \psi(N), \frac{1}{12} \psi(N)$, namely the cusp $x_{N}=\infty$ (i.e., the distinguished cusp $\tau=0$ );

(2) $\sigma_{\infty}(N)-1$ singular points with exponents 0,0 , namely the remaining cusps, including $x_{N}=0$ (i.e., the distinguished cusp $\tau=\mathrm{i} \infty$ );

(3) $\epsilon_{\mathrm{i}}(N)$ singular points with exponents $0, \frac{1}{2}$, namely the order-2 elliptic fixed points;

(4) $\epsilon_{\rho}(N)$ singular points with exponents $0, \frac{1}{3}$, namely the order-3 elliptic fixed points.

This weak lifting is obtained from the lifting prefactor $\tilde{f}_{e}\left(x_{N}\right)=P_{N}\left(x_{N}\right)^{-1 / 12}$.

Any ratio of independent local solutions of $\tilde{M}_{N} \tilde{u}=0$ equals $(a \tau+b) /(c \tau+d)$ for some $a, b, c, d$ with $a d \neq b c$. The unique local solution of $\tilde{M}_{N} \tilde{u}=0$ analytic at the distinguished cusp $x_{N}=0$ (i.e., at $\tau=\mathrm{i} \infty$ ) and equaling 1 there will be $h_{N}$.

Remark 4.8.1. The differential equation $\tilde{M}_{N} \tilde{u}=0$ satisfies the conditions of Theorem 4.2. By Theorem 4.3(ii), it is uniquely characterized by the given list of singular points and exponents, and the fact that any ratio of independent local solutions is of the form $(a \tau+b) /(c \tau+d)$. Its two-dimensional space of solutions, viewed as functions of $\tau$, is $h_{N}\left(x_{N}(\tau)\right)(\mathbb{C} \tau+\mathbb{C})$.

Proof of Proposition 4.8. This is an application of Lemma 4.6, with $\alpha_{\infty}=\frac{1}{12}$. The given exponents are computed thus. Suppose the prefactor were absent, i.e., suppose $\tilde{u}(\cdot)=u(\xi(\cdot))$. Then above $\hat{J}=0$, each cusp on $X_{0}(N)$ would have exponents 0,0 , irrespective of its multiplicity. A point on the fibre above $\hat{J}=1$ would have exponents $0, \frac{1}{2}$ if it has unit multiplicity, i.e., if it is an order-2 elliptic point, and exponents 0,1 otherwise, indicating it would be an ordinary (non-singular) point. The simple roots of $P_{N}\left(x_{N}\right)$ are the $\varepsilon_{\rho}(N)$ order-3 elliptic points on the $x_{N}$-sphere, and all other roots are triple. For a lifting, the exponents at these two sorts of point would be $\frac{1}{12}, \frac{5}{12}$ and $\frac{1}{4}, \frac{5}{4}$. Including the prefactor $P_{N}\left(x_{N}\right)^{-1 / 12}$ shifts them to $0, \frac{1}{3}$ and 0,1 ; so the latter will no longer be singular points. It also shifts the exponents at the cusp $x_{N}=\infty$ from 0,0 to $\frac{1}{12} \operatorname{deg} P_{N}, \frac{1}{12} \operatorname{deg} P_{N}$. 
The weak-lifted differential equation $\tilde{M}_{N} \tilde{u}=0$ of the proposition, which is of the form $\left[D_{x_{N}}^{2}+\tilde{\mathcal{A}} \cdot D_{x_{N}}+\tilde{\mathcal{B}}\right] \tilde{u}=0$ for certain $\tilde{\mathcal{A}}, \tilde{\mathcal{B}} \in \mathbb{Q}\left(x_{N}\right)$, can readily be derived from ${ }_{2} \mathcal{E}_{1}\left(\frac{1}{12}, \frac{5}{12} ; 1\right)$ by changing variables. Like ${ }_{2} \mathcal{E}_{1}\left(\frac{1}{12}, \frac{5}{12} ; 1\right)$, it is always based on an operator of the normal form (4.2). The curve $X_{0}(N)$ is of genus zero only if $N=2,3,4,5,6,7,8,9,10,12,13,16,18,25$, and the number of singular points, namely $m(N):=\left(\sigma_{\infty}+\varepsilon_{\mathrm{i}}+\varepsilon_{\rho}\right)(N)$, is $3,3,3,4,4,4,4,4,6,6,6,6,8,8$, respectively. So when $N=2,3,4$, the equation $\tilde{M}_{N} \tilde{u}=0$ is of hypergeometric type on the $x_{N}$-sphere. (Two of its singular points are at the cusps $x_{N}=0, \infty$, so a linear scaling of $x_{N}$ will reduce it to the Gauß form.) When $N=5,6,7,8,9$, it is of Heun type, and its solutions, including $h_{N}$, may be expressed in terms of the local Heun function $\mathrm{Hl}$ [26]. In general, the coefficients $\left\{c_{n}^{(N)}\right\}_{n=0}^{\infty}$ of the power series expansion $h_{N}\left(x_{N}\right)=\sum_{n=0}^{\infty} c_{n}^{(N)} x_{N}^{n}$ will satisfy an $[m(N)-1]$-term recurrence relation.

Proposition 4.9. The fundamental analytic function $h_{N}$ defined by (4.4) when $X_{0}(N)$ is of genus zero, on a neighborhood of the point $x_{N}=0$ on $X_{0}(N)$, extends by continuation to a weight-1 modular form for $\Gamma_{0}(N)$ on $\mathfrak{H}^{*} \ni \tau$, with some multiplier system. One may write

$$
h_{N}\left(x_{N}(\tau)\right)=P_{N}(0)^{1 / 12} Q_{N}\left(x_{N}(\tau)\right)^{-1 / 12} \eta^{2}(\tau),
$$

where $\eta(\cdot)$ is the Dedekind eta function. This modular form is regular and nonvanishing at each cusp of $\Gamma_{0}(N)$ in $\mathbb{P}^{1}(\mathbb{Q}) \ni \tau$ other than those in the equivalence class $\left[\frac{1}{1}\right]_{N} \ni 0$, at each of which its order is $\psi(N) /(12 N)$.

Proof. Stiller 32 shows that in a neighborhood of $\tau=\mathrm{i} \infty$, where $\hat{J}=\hat{J}(\tau)$ equals zero, the analytic solution ${ }_{2} F_{1}\left(\frac{1}{12}, \frac{5}{12} ; 1 ; \hat{J}\right)$ of ${ }_{2} \mathcal{E}_{1}\left(\frac{1}{12}, \frac{5}{12} ; 1\right)$, regarded like $\hat{J}$ as a function of $\tau$, equals $12^{1 / 4} \eta^{2}(\tau) \hat{J}^{-1 / 12}$, a weight- 1 modular form for $\Gamma$. Equivalently,

$$
\eta^{2}(\tau)=12^{-1 / 4} \hat{J}_{2}^{1 / 12} F_{1}\left(\frac{1}{12}, \frac{5}{12} ; 1 ; \hat{J}\right),
$$

with the root $\hat{J}^{1 / 12}$ taken positive when $\hat{J}>0$. (The identity (4.6) can be traced back to Dedekind, who at one point defined $\eta^{2}(\tau)$ as an expression of hypergeometric type equal to the right-hand side [8, p. 137].) Combining this fact with $\hat{J}\left(x_{N}\right)=1728 Q_{N}\left(x_{N}\right) / P\left(x_{N}\right)$ and (4.4) yields (4.5).

$\eta(\tau), x_{N}(\tau)$ are of weight $1 / 2,0$, and the $Q_{N}$ factor in (4.5) is zero only at cusps of $\mathfrak{H}^{*}$, so the right-hand side of (4.5) is holomorphic on $\mathfrak{H}$ and is a weight-1 modular form. The roots of the polynomial $Q_{N}\left(x_{N}\right)$ are bijective with the cusp equivalence classes $\left[\frac{a}{d}\right]_{N}$ of $X_{0}(N)$ other than $\left[\frac{1}{1}\right]_{N}$, at which $x_{N}=\infty$. Any root of $Q_{N}$ has multiplicity equal to the cusp width $e_{d, N}$, i.e., the multiplicity with which $\left[\frac{a}{d}\right]_{N}$ is mapped to $X(1)$. Also, the order of $\eta(\tau)$ at any cusp $\tau \in \mathbb{P}^{1}(\mathbb{Q})$ equals $1 / 24$. So altogether, the order of $h_{N}\left(x_{N}(\tau)\right)$ at any cusp in $\left[\frac{a}{d}\right]_{N} \neq\left[\frac{1}{1}\right]_{N}$ will equal $(-1 / 12) e_{d, N} / e_{d, N}+2 \cdot \frac{1}{24}=0$.

The cusp equivalence class $\left[\frac{1}{1}\right]_{N}$, on which $x_{N}=\infty$, is best handled by referring to the original definition (4.4) of $h_{N}$. Since $\operatorname{deg} P_{N}=\psi(N)$, the order of $h_{N}\left(x_{N}(\tau)\right)$ at any cusp in $\left[\frac{1}{1}\right]_{N}$ will be $(1 / 12) \psi(N) / e_{1, N}=\psi(N) /(12 N)$.

For each of $N=2, \ldots, 7$, an eta-product representation for the weight-1 form $h_{N}\left(x_{N}(\tau)\right)$ can be computed from (4.5), and the formula $j=P_{N}\left(x_{N}\right) / Q_{N}\left(x_{N}\right)$ and eta-product representation for $x_{N}=x_{N}(\tau)$ given in the appendix. These are listed in Table 1. The multiplier systems of the $h_{N}\left(x_{N}(\tau)\right)$ are non-trivial but are 
TABLE 1. The Hauptmodul $x_{N}$ and weight-1 modular form $h_{N}$ for $\Gamma_{0}(N)$, as eta products. (For notation see the appendix.)

\begin{tabular}{|c|l|l|}
\hline$N$ & \multicolumn{1}{|c|}{$x_{N}(\tau)$} & \multicolumn{1}{c|}{$h_{N}\left(x_{N}(\tau)\right)$} \\
\hline 2 & $2^{12} \cdot[2]^{24} /[1]^{24}$ & {$[1]^{4} /[2]^{2}$} \\
3 & $3^{6} \cdot[3]^{12} /[1]^{12}$ & {$[1]^{3} /[3]$} \\
4 & $2^{8} \cdot[4]^{8} /[1]^{8}$ & {$[1]^{4} /[2]^{2}$} \\
5 & $5^{3} \cdot[5]^{6} /[1]^{6}$ & $\left\{[1]^{5} /[5]\right\}^{1 / 2}$ \\
6 & $2^{3} 3^{2} \cdot[2][6]^{5} /[1]^{5}[3]$ & {$[1]^{6}[6] /[2]^{3}[3]^{2}$} \\
7 & $7^{2} \cdot[7]^{4} /[1]^{4}$ & $\left\{[1]^{7} /[7]\right\}^{1 / 3}$ \\
\hline
\end{tabular}

not difficult to compute. For example, $h_{6}\left(x_{6}(\tau)\right)$ is of quadratic Nebentypus: its transformation under any $\left(\begin{array}{ll}a & b \\ c & d\end{array}\right) \in \Gamma_{0}(6)$ includes a $( \pm 1)$-valued factor equal to the Dirichlet character $\chi(d):=\left(\frac{d}{6}\right)$, where $(\dot{)})$ is the Jacobi symbol. (Cf. [33, $\left.\S 2.2\right]$.)

The new interpretation of $h_{N}$ makes contact with the theory of differential equations satisfied by modular forms, originating with Stiller 31. If $\Gamma_{1} \leq \Gamma$ is a congruence subgroup of genus zero with Hauptmodul $x$, then any weight- $k$ modular form $f$ for $\Gamma_{1}$ that has a power series expansion $f=\sum_{n=0}^{\infty} c_{n} x^{n}$ and can be viewed locally (near $x=0$ ) as a function of $x$ will necessarily satisfy an order- $(k+1)$ differential equation with respect to $x$, which can be constructed algorithmically. Applied to any $h_{N}$ viewed as a weight-1 modular form for $\Gamma_{0}(N)$, the algorithm will recover the weak-lifted second-order equation $\tilde{M}_{N} \tilde{u}=0$ of Proposition 4.8 .

We shall not attempt here to define an analogue of the modular form $h_{N}\left(x_{N}(\tau)\right)$ when $X_{0}(N)$ is of positive genus. Although the curves $X_{0}(36), X_{0}(49)$ are of genus 1 , it will nonetheless prove possible in $\S \S[$ and 6 to derive hypergeometric identities from the way in which they cover $X_{0}(6), X_{0}(7)$, at the price of some awkwardness.

\section{Key RESUlts}

It can now be explained how hypergeometric identities follow from the covering maps $\phi_{N}: X_{0}\left(N^{2}\right) \rightarrow X_{0}(N)$ and $\phi_{N}^{\prime}: X_{0}\left(N^{2}\right) \rightarrow X_{0}(N)^{\prime}$. These coverings are induced by the subgroup relations $\Gamma_{0}\left(N^{2}\right)<\Gamma_{0}(N)$ and $\Gamma_{0}\left(N^{2}\right)<\Gamma_{0}(N)^{\prime}$. Note that the map $\phi_{N}$ also has an obvious elliptic-curve interpretation: it acts on $X_{0}\left(N^{2}\right)$, the space of isomorphism classes of $N^{2}$-isogenies between elliptic curves $E, E^{\prime}=E / C_{N^{2}}$, by replacing $C_{N^{2}}$ by its $C_{N}$ subgroup. The map $\phi_{N}^{\prime}$ acts on the dual $N$-isogeny between $E^{\prime}, E=E^{\prime} / C_{N^{2}}$ in the same way.

If $X_{0}(N)$ is of genus zero and has a canonical Hauptmodul $x_{N}$ with divisor $(\mathrm{i} \infty)-(0)$ as reviewed in the appendix, then the fundamental weight-1 modular form $h_{N}$ is given near the point $x_{N}=0$, i.e., the cusp $\tau=\mathrm{i} \infty$, by Definition 4.7 . Consider first the case when $X_{0}\left(N^{2}\right)$ too is of genus zero, with Hauptmodul $x_{N^{2}}$, and a corresponding weight-1 modular form $h_{N^{2}}$ defined near $x_{N^{2}}=0$ (which is the same cusp $\tau=\mathrm{i} \infty$ on $\left.\mathfrak{H}^{*}\right)$.

Both $x_{N}$ and $x_{N}^{\prime}$, the canonical Hauptmodul on $X_{0}(N)^{\prime}$, will be rational functions of $x_{N^{2}}$. That is, $x_{N}=R\left(x_{N^{2}}\right) / S\left(x_{N^{2}}\right)$ and $x_{N}^{\prime}=R^{\prime}\left(x_{N^{2}}\right) / S^{\prime}\left(x_{N^{2}}\right)$ for certain $R, S, R^{\prime}, S^{\prime} \in \mathbb{Q}\left[x_{N^{2}}\right]$. (Primes do not indicate derivatives.) Both rational functions will have mapping degree equal to $N$, the index of $\Gamma_{0}\left(N^{2}\right)$ in $\Gamma_{0}(N)$ or $\Gamma_{0}(N)^{\prime}$. 
By Lemma 3.1, the fibre of $\phi_{N}$ above $x_{N}=\infty$, i.e., above the cusp $\left[\frac{1}{1}\right]_{N}$ of $X_{0}(N)$ containing $\tau=0$, consists of the point $x_{N^{2}}=\infty$, i.e., the cusp $\left[\frac{1}{1}\right]_{N^{2}}$ of $X_{0}\left(N^{2}\right)$, with multiplicity $N$. So $\operatorname{deg} R=N$ and $\operatorname{deg} S=0$; one may take $S\left(x_{N^{2}}\right):=1$. Also by Lemma 3.1 the fibre of $\phi_{N}^{\prime}$ above $x_{N}^{\prime}=0$, i.e., above the cusp $\left[\frac{1}{N}\right]_{N} / N$ of $X_{0}(N)^{\prime}$, consists of the point $x_{N^{2}}=0$, i.e., the cusp $\left[\frac{1}{N^{2}}\right]_{N^{2}}$, with multiplicity $N$. Moreover, the fibre of $\phi_{N}^{\prime}$ above $x_{N}^{\prime}=\infty$, i.e., above $\left[\frac{1}{1}\right]_{N} / N$, contains the point $x_{N^{2}}=\infty$, i.e., the cusp $\left[\frac{1}{1}\right]_{N^{2}}$, with multiplicity 1. So $R^{\prime}\left(x_{N^{2}}\right) \propto x_{N^{2}}^{N}$ and $\operatorname{deg} S^{\prime}=N-1$; one may take $R^{\prime}\left(x_{N^{2}}\right):=x_{N^{2}}^{N}$. It turns out that the two remaining polynomials $R, S^{\prime}$ are always monic and in $\mathbb{Z}\left[x_{N^{2}}\right]$, but that fact will not be needed.

Theorem 5.1. If $X_{0}(N)$ and $X_{0}\left(N^{2}\right)$ are of genus zero (i.e., if $\left.N=2,3,4,5\right)$, then the hypergeometric identity

$$
h_{N^{2}}\left(x_{N^{2}}\right)=h_{N}\left(R\left(x_{N^{2}}\right)\right)=\left[S^{\prime}\left(x_{N^{2}}\right) / S^{\prime}(0)\right]^{-\psi(N) / 12} h_{N}\left(\frac{x_{N^{2}}^{N}}{S^{\prime}\left(x_{N^{2}}\right)}\right)
$$

holds in a neighborhood of the point $x_{N^{2}}=0$ on $X_{0}\left(N^{2}\right)$, i.e., of the cusp $\tau=\mathrm{i} \infty$.

Remark 5.1.1. The first equality says that as functions of $\tau \in \mathfrak{H}^{*}$, the modular forms $h_{N^{2}}\left(x_{N^{2}}(\tau)\right)$ and $h_{N}\left(x_{N}(\tau)\right)$ are equal. This is confirmed in the $N=2$ case by Table 1 and is easily seen to be true of the other genus-zero cases $N=3,4,5$.

Proof. ${ }_{2} \mathcal{E}_{1}\left(\frac{1}{12}, \frac{5}{12} ; 1\right)$ is weak-lifted by $\pi_{N}$ to $\tilde{M}_{N} \tilde{u}=0$ and by $\pi_{N^{2}}$ to $\tilde{M}_{N^{2}} \tilde{u}=0$. The unique (normalized) analytic local solutions at $x_{N}=0$, resp. $x_{N^{2}}=0$, are $h_{N}$ and $h_{N^{2}}$. The expression $h_{N}(R(\cdot))$ is the unique (normalized) analytic local solution at $x_{N^{2}}=0$ of what may be denoted $\tilde{\tilde{M}}_{N^{2}} \tilde{u}=0$, the lifting (not merely weak lifting) of $\tilde{M}_{N} \tilde{u}=0$ from $X_{0}(N)$ to $X_{0}\left(N^{2}\right)$, along $\phi_{N}$. Since $\pi_{N^{2}}=\pi_{N} \circ \phi_{N}$, the equation $\tilde{\tilde{M}}_{N^{2}} \tilde{u}=0$ like $\tilde{M}_{N^{2}} \tilde{u}=0$ is a weak lifting of ${ }_{2} \varepsilon_{1}\left(\frac{1}{12}, \frac{5}{12} ; 1\right)$ to $X_{0}\left(N^{2}\right)$, along $\pi_{N^{2}}$. But $\tilde{M}_{N^{2}}, \tilde{\tilde{M}}_{N^{2}}$ have the same exponents: e.g., $N\left(\frac{1}{12} \psi(N), \frac{1}{12} \psi(N)\right)=$ $\left(\frac{1}{12} \psi\left(N^{2}\right), \frac{1}{12} \psi\left(N^{2}\right)\right)$ at the cusp $x_{N^{2}}=\infty$, and 0,0 at each of the other cusps. So by Theorem 4.3 (ii) they are the same, and $h_{N}(R(\cdot))$ must equal $h_{N^{2}}(\cdot)$.

To show the second expression also equals $h_{N^{2}}\left(x_{N^{2}}\right)$, consider $\tilde{M}_{N}^{\prime} \tilde{u}=0$, the Fuchsian equation on the $x_{N}^{\prime}$-sphere $X_{0}(N)^{\prime}$ obtained by formally substituting $x_{N}^{\prime}$ for $x_{N}$ in $\tilde{M}_{N} \tilde{u}=0$, and its lifting $\tilde{M}_{N^{2}}^{\prime} \tilde{u}=0$ to $X_{0}\left(N^{2}\right)$, along $\phi_{N}^{\prime}$. Any ratio of independent local solutions of $\tilde{M}_{N} \tilde{u}=0$ is of the form $(a \tau+b) /(c \tau+d)$. Since $x_{N}^{\prime}(\tau):=x_{N}(N \tau)$, the same is true of $\tilde{M}_{N}^{\prime} \tilde{u}=0$. (The coefficients $a, c$ are multiplied by $N$.) By the definition of a lifting, it is also true of $\tilde{\tilde{M}}_{N^{2}}^{\prime} \tilde{u}=0$, and by Proposition 4.8 it happens to be true of $\tilde{M}_{N^{2}} \tilde{u}=0$, as well. So $\tilde{M}_{N^{2}}, \tilde{\tilde{M}}_{N^{2}}^{\prime}$ are projectively the same: they determine the same flat connection on the $\mathbb{P}^{1}(\mathbb{C})$-bundle over $X_{0}\left(N^{2}\right)$. They necessarily have the same exponent differences.

To make $\tilde{\tilde{M}}_{N^{2}}^{\prime}$ identical to $\tilde{M}_{N^{2}}$, it may be redefined as a weak lifting, incorporating a prefactor that shifts the exponents at each singular point to those of $\tilde{M}_{N^{2}}$. (By Theorem 4.3(ii), that will suffice.) Only the exponents at the cusps of $X_{0}\left(N^{2}\right)$ need to be altered. As originally defined, $\tilde{\tilde{M}}_{N^{2}}^{\prime}$ had exponents $n\left(\frac{1}{12} \psi(N), \frac{1}{12} \psi(N)\right)$ at each cusp in the fibre of $\phi_{N}^{\prime}$ above $x_{N}^{\prime}=\infty$, where $n$ is the multiplicity with which the cusp appears. Letting the lifting prefactor be $S^{\prime}\left(x_{N^{2}}\right)^{-\psi(N) / 12}$, which is a member of the collection of algebraic functions on the upper curve $X_{0}\left(N^{2}\right)$ denoted $\tilde{\mathbb{K}}_{e}^{\times}$ in the last section, will shift the exponents at all cusps other than $x_{N^{2}}=\infty$ to 0,0 
as desired. So with this choice, $\tilde{\tilde{M}}_{N^{2}}^{\prime}$ will equal $\tilde{M}_{N^{2}}$, and the second expression of the theorem, which is the unique (normalized) analytic local solution of $\tilde{\tilde{M}}_{N^{2}}^{\prime} \tilde{u}=0$ at $x_{N^{2}}=0$, will equal $h_{N^{2}}\left(x_{N^{2}}\right)$, the corresponding local solution of $\tilde{M}_{N^{2}} \tilde{u}=0$.

If $X_{0}\left(N^{2}\right)$ unlike $X_{0}(N)$ is of positive genus, then it will have no Hauptmodul $x_{N^{2}}$. In this case Theorem 5.1 must be replaced by Theorem 5.3, which actually subsumes it, though it is less explicit and must be supplemented by the subsequent proposition. But what it says about the case $g\left(X_{0}\left(N^{2}\right)\right)>0$ is significantly weaker than Theorem [5.1] If the genus is positive, Theorem 4.3(ii) is not available, and it is consequently difficult to rule out the possibility that the two sides of the hypergeometric 'identity' may in fact differ, due to their being solutions of Fuchsian differential equations with different values for their affine accessory parameters. In the cases $N=6,7$ treated in the next section, this will fortunately not be an issue, since the two differential equations on $X_{0}\left(N^{2}\right)$ can be computed explicitly and shown to be identical. But in Theorem 5.3, the following ad hoc notion of equivalence will be used. Note that if $g\left(X_{0}\left(N^{2}\right)\right)=0, \mathfrak{h}^{(1)} \sim \mathfrak{h}^{(2)}$ implies $\mathfrak{h}^{(1)}=\mathfrak{h}^{(2)}$.

Definition 5.2. If $\mathfrak{h}^{(1)}, \mathfrak{h}^{(2)}$ are analytic functions in a neighborhood of the cusp $\left[\frac{1}{N^{2}}\right]$ on $X_{0}\left(N^{2}\right)$, i.e., of the point $\tau=\mathfrak{i} \infty, \mathfrak{h}^{(1)} \sim \mathfrak{h}^{(2)}$ signifies that they are the unique (normalized) analytic solutions there of $L_{1} \mathfrak{h}=0, L_{2} \mathfrak{h}=0$, two Fuchsian differential equations satisfying the following conditions. (i) Each satisfies the conditions of Theorem 4.2, any ratio of independent solutions is of the form $(a \tau+b) /(c \tau+d)$, the singular points are the fixed points of $\Gamma_{0}\left(N^{2}\right)$, and the exponent differences are $1 / k$ at each fixed point of order $k$ and zero at each cusp.

(ii) The exponents (not merely exponent differences) of $L_{1}, L_{2}$ are the same.

Theorem 5.3. Suppose that $X_{0}(N)$ is of genus zero, and that $f_{N^{2}}$ is an element of the function field of $X_{0}\left(N^{2}\right)$ that satisfies the divisor condition

$$
\begin{aligned}
\operatorname{div}\left(f_{N^{2}}\right) & =\psi(N) \cdot\left[\left(\phi_{N}^{\prime}\right)^{*}\left(\left[\frac{1}{1}\right]_{N} / N\right)-\left(\phi_{N}\right)^{*}\left(\left[\frac{1}{1}\right]_{N}\right)\right], \\
& =\psi(N) \cdot \sum_{\substack{d \text { s.t. } d|N| N^{2}, a=a_{1}, \ldots, a_{\varphi(f)}}}\left[\left(\left[\frac{a}{d}\right]_{N^{2}}\right)-\left(\left[\frac{1}{1}\right]_{N^{2}}\right)\right],
\end{aligned}
$$

where $\left(\phi_{N}\right)^{*},\left(\phi_{N}^{\prime}\right)^{*}$ lift divisors on $X_{0}(N), X_{0}(N)^{\prime}$ to those on $X_{0}\left(N^{2}\right)$, and where the second equality comes from Lemma 3.1. Then the hypergeometric 'identity'

$$
h_{N}\left(\phi_{N}(\cdot)\right) \sim\left[f_{N^{2}}(\cdot) / f_{N^{2}}\left(\left[\frac{1}{N^{2}}\right]_{N^{2}}\right)\right]^{-1 / 12} h_{N}\left(\phi_{N}^{\prime}(\cdot)\right)
$$

holds in a neighborhood of the cusp $\left[\frac{1}{N^{2}}\right]$ on $X_{0}\left(N^{2}\right)$, i.e., of the point $\tau=\mathrm{i} \infty$.

Proof. As in the last proof, consider $\tilde{\tilde{M}}_{N^{2}}, \tilde{\tilde{M}}_{N^{2}}^{\prime}$, the liftings of $\tilde{M}_{N}, \tilde{M}_{N}^{\prime}$ to $X_{0}\left(N^{2}\right)$ along $\phi_{N}, \phi_{N}^{\prime}$. They are projectively the same, in the sense that any ratio of independent local solutions of $\tilde{\tilde{M}}_{N^{2}} \tilde{u}=0$, and also of $\tilde{\tilde{M}}_{N^{2}}^{\prime} \tilde{u}=0$, is of the form $(a \tau+b) /(c \tau+d)$. So they have the same exponent differences at each point of $X_{0}\left(N^{2}\right)$. But they have different exponents. At any cusp of $X_{0}\left(N^{2}\right)$ above $x_{N}=\infty$, resp. $x_{N}^{\prime}=\infty$, i.e., above $\left[\frac{1}{1}\right]_{N}$, resp. $\left[\frac{1}{1}\right]_{N} / N$, the exponents of $\tilde{\tilde{M}}_{N^{2}}$, resp. $\tilde{\tilde{M}}_{N^{2}}^{\prime}$, are $n\left(\frac{1}{12} \psi(N), \frac{1}{12} \psi(N)\right)$, where $n$ is the cusp multiplicity. Converting $\tilde{\tilde{M}}_{N^{2}}^{\prime}$ to a weak lifting by including a lifting prefactor $f_{N^{2}}^{-1 / 12}$, where $f_{N^{2}}$ satisfies the divisor condition of the theorem, will ensure that $\tilde{\tilde{M}}_{N^{2}}$ and $\tilde{\tilde{M}}_{N^{2}}^{\prime}$ have the same 
exponents. The two sides of (5.1) are their respective unique (normalized) analytic local solutions in a neighborhood of the cusp $\left[\frac{1}{N^{2}}\right]_{N^{2}}$.

The divisor of the theorem is principal, i.e., such a function $f_{N^{2}}$ always exists. The following proposition supplies an explicit formula for it.

Proposition 5.4. The automorphic function $f_{N^{2}}: X_{0}\left(N^{2}\right) \rightarrow \mathbb{P}^{1}(\mathbb{C})$ of the previous theorem can be chosen to be

$$
f_{N^{2}}(\cdot)=\prod_{\substack{d \text { s.t. } 1<d \mid N, a=a_{1}, \ldots, a_{\varphi\left(f_{d, N}\right)}}} \frac{\left[x_{N}\left(\phi_{N}(\cdot)\right)-x_{N}\left(\left[\frac{a}{d}\right]_{N}\right)\right]^{e_{N} / d, N}}{\left[x_{N}^{\prime}\left(\phi_{N}^{\prime}(\cdot)\right)-x_{N}\left(\left[\frac{a}{d}\right]_{N}\right)\right]^{e_{d, N}}}
$$

a product over the $\sigma_{\infty}(N)-1$ cusps of $X_{0}(N)$ other than $\left[\frac{1}{1}\right]_{N}$, i.e., other than $\tau=0$. Here $e_{d, N}:=N / d(d, N / d)$ is the width of the cusp $\left[\frac{a}{d}\right]_{N}$, as above.

Proof. On $X_{0}(N)$, the function $x_{N}-x_{N}\left(\left[\frac{a}{d}\right]_{N}\right)$ has divisor $\left(\left[\frac{a}{d}\right]_{N}\right)-\left(\left[\frac{1}{1}\right]_{N}\right)$, the latter term coming from the pole of $x_{N}$ at $\tau=0$. Similarly on $X_{0}(N)^{\prime}$, the function $x_{N}^{\prime}-x_{N}\left(\left[\frac{a}{d}\right]_{N}\right)$ has divisor $\left(\left[\frac{a}{d}\right]_{N} / N\right)-\left(\left[\frac{1}{1}\right]_{N} / N\right)$. So on $X_{0}\left(N^{2}\right), f_{N^{2}}$ has divisor $\sum_{\substack{d \text { s.t. } d \mid N, a=a_{1}, \ldots, a_{\varphi\left(f_{d, N}\right)}}}\left[e_{N / d, N} \cdot\left(\phi_{N}\right)^{*}\left(\left[\frac{a}{d}\right]_{N}-\left[\frac{1}{1}\right]_{N}\right)-e_{d, N} \cdot\left(\phi_{N}^{\prime}\right)^{*}\left(\left[\frac{a}{d}\right]_{N} / N-\left[\frac{1}{1}\right]_{N} / N\right)\right]$,

where the restriction to $d>1$ has been innocuously dropped. This expression splits naturally into two sub-expressions, the first of which is

$$
\begin{array}{r}
\sum_{\substack{d \text { s.t. } d \mid N, a=a_{1}, \ldots, a_{\varphi}\left(f_{d, N}\right)}}\left[e_{d, N} \cdot\left(\phi_{N}^{\prime}\right)^{*}\left(\left[\frac{1}{1}\right]_{N} / N\right)-e_{N / d, N} \cdot\left(\phi_{N}\right)^{*}\left(\left[\frac{1}{1}\right]_{N}\right)\right] \\
=\psi(N) \cdot\left[\left(\phi_{N}^{\prime}\right)^{*}\left(\left[\frac{1}{1}\right]_{N} / N\right)-\left(\phi_{N}\right)^{*}\left(\left[\frac{1}{1}\right]_{N}\right)\right] .
\end{array}
$$

Each cusp of the form $\left[\frac{a}{d}\right]_{N}$ has ramification index $e_{d, N}$ over the unique cusp of $X(1)$, so the sum $\sum e_{d, N}$ over all $\sigma_{\infty}(N)$ cusps must equal $\psi(N)$, the degree of the covering map, as must $\sum e_{N / d, N}$, by symmetry, which explains the equality between (5.3a) and (5.3b). The divisor (5.3b) is identical to the desired divisor of Theorem 5.3, so it remains to show that the second sub-expression, namely

$$
\sum_{\substack{d \text { s.t. } d \mid N, a=a_{1}, \ldots, a_{\varphi}\left(f_{d, N}\right)}}\left[e_{N / d, N} \cdot\left(\phi_{N}\right)^{*}\left(\left[\frac{a}{d}\right]_{N}\right)\right]-\sum_{\substack{d \text { s.t. } d \mid N, a=a_{1}, \ldots, a_{\varphi}\left(f_{d, N}\right)}}\left[e_{d, N} \cdot\left(\phi_{N}^{\prime}\right)^{*}\left(\left[\frac{a}{d}\right]_{N} / N\right)\right],
$$

equals zero. The first term can be viewed as a sum over the $\sigma_{\infty}\left(N^{2}\right)$ cusps of $X_{0}\left(N^{2}\right)$. Any cusp $\left[\frac{\tilde{a}}{d}\right]_{N^{2}}$ with $\tilde{d} \mid N^{2}$ that is sent to a cusp of the form $\left[\frac{a}{d}\right]_{N}$ by $\phi_{N}$ will appear in this sum with multiplicity equal to $e_{N / d, N}=d /(d, N / d)$ times its ramification index, which by Lemma 3.2 equals $e_{d, N^{2}} / e_{d, N}=N(d, N / d) / d$. So each of the $\sigma_{\infty}\left(N^{2}\right)$ cusps of $X_{0}\left(N^{2}\right)$ appears with multiplicity $e_{N / d, N} e_{d, N^{2}} / e_{d, N}=N$. The subtrahend can be obtained from the first term by applying the Fricke involution $w_{N^{2}}$, which interchanges each cusp of the form $\left[\frac{\tilde{a}}{\tilde{d}}\right]_{N^{2}}$ with a cusp of the form $\left[\frac{\tilde{a}^{\prime}}{N^{2} / d}\right]_{N^{2}}$. So it equals the first term, and their difference equals zero. 


\section{Explicit formulas: The CASes $N=2, \ldots, 7$}

6.1. The $g\left(X_{0}(N)\right)=0, g\left(X_{0}\left(N^{2}\right)\right)=0$ cases $(N=2,3,4,5)$. When $N$ equals $2,3,4$, or 5 , the fundamental weight- 1 modular form $h_{N}$ for $\Gamma_{0}(N)$ can be expressed in terms of ${ }_{2} F_{1}$ on a neighborhood of the point $x_{N}=0$ of $X_{0}(N)$ by

$$
\begin{aligned}
h_{2}(z) & =\left[\frac{1}{16^{3}}(z+16)^{3}\right]_{2}^{-1 / 12}{ }_{2} F_{1}\left(\frac{1}{12}, \frac{5}{12} ; 1 ; \frac{1728 z}{(z+16)^{3}}\right) \\
& ={ }_{2} F_{1}\left(\frac{1}{4}, \frac{1}{4} ; 1 ;-z / 64\right) ; \\
h_{3}(z) & =\left[\frac{1}{3^{6}}(z+3)^{3}(z+27)\right]^{-1 / 12}{ }_{2} F_{1}\left(\frac{1}{12}, \frac{5}{12} ; 1 ; \frac{1728 z}{(z+3)^{3}(z+27)}\right) \\
& ={ }_{2} F_{1}\left(\frac{1}{3}, \frac{1}{3} ; 1 ;-z / 27\right) ; \\
h_{4}(z) & =\left[\frac{1}{16^{3}}\left(z^{2}+16 z+16\right)^{3}\right]^{-1 / 12}{ }_{2} F_{1}\left(\frac{1}{12}, \frac{5}{12} ; 1 ; \frac{1728 z(z+16)}{\left(z^{2}+16 z+16\right)^{3}}\right) \\
& ={ }_{2} F_{1}\left(\frac{1}{2}, \frac{1}{2} ; 1 ;-z / 16\right) ; \\
h_{5}(z) & =\left[\frac{1}{5^{3}}\left(z^{2}+10 z+5\right)^{3}\right]^{-1 / 12}{ }_{2} F_{1}\left(\frac{1}{12}, \frac{5}{12} ; 1 ; \frac{1728 z}{\left(z^{2}+10 z+5\right)^{3}}\right),
\end{aligned}
$$

where $z$ signifies $x_{N}$. The formulas (6.1a), 66.2a), 66.3a), 6.4a) follow from Definition 4.7 and the formulas of the form $j=P_{N}\left(x_{N}\right) / Q_{N}\left(x_{N}\right)$ for the covering $\pi_{N}: X_{0}(N) \rightarrow X(1) \cong \mathbb{P}^{1}(\mathbb{C}) \ni j$ given in the appendix. The simpler expressions (6.1b), 6.2b) 6.6. 6ollow from the fact mentioned after Proposition 4.8. each of $h_{2}, h_{3}, h_{4}$ satisfies an equation of hypergeometric type. For instance, lifting ${ }_{2} \mathcal{E}_{1}\left(\frac{1}{2}, \frac{5}{12} ; 1\right)$ from the $\hat{J}_{\text {-sphere }} X(1)$ to $X_{0}(2) \ni x_{2}=: z$ yields

$$
\left\{D_{z}^{2}+\left[\frac{1}{z}+\frac{1}{2(z+64)}\right] D_{z}+\left[\frac{1}{16 z(z+64)}\right]\right\} h_{2}=0
$$

This is reduced to the Gauß equation ${ }_{2} \mathcal{E}_{1}\left(\frac{1}{4}, \frac{1}{4} ; 1\right)$ by the linear scaling $\hat{z}=-z / 64$, yielding (6.1b). The expressions (6.2b), (6.3b) follow likewise. The equivalence of the 'a' and 'b' expressions yields a cubic, a quartic, and a sextic transformation of ${ }_{2} F_{1}$. These turn out to be special cases of three of Goursat's hypergeometric transformations, listed as (121), (129), (135) in his classical tabulation [17.

There is no expression (6.4b), since the Fuchsian equation obtained by lifting ${ }_{2} \mathcal{E}_{1}\left(\frac{1}{2}, \frac{5}{12} ; 1\right)$ to $X_{0}(5) \ni x_{5}=: z$, which appears as (1.4) above, has four singular points rather than three. Its analytic solution $h_{5}$ near $z=0$ can be reduced to $\mathrm{Hl}$ by an appropriate Möbius transformation $\hat{z}=(A z+B) /(C z+D)$. By definition, $\mathrm{Hl}(a, q ; \alpha, \beta, \gamma, \delta ; \cdot)$ is the unique (normalized) analytic local solution at $\hat{z}=0$ of a canonical Fuchsian equation with the four singular points $\hat{z}=0,1, a, \infty$. (The parameters $\alpha, \beta, \gamma, \delta$ are exponent-related; $q$ is the accessory parameter.) So the transformation $\left(\begin{array}{ll}A & B \\ C\end{array}\right) \in P G L(2, \mathbb{C})$ must take the singular points $0,-11 \pm 2 \mathrm{i}, \infty$ of (1.4) to $0,1, a, \infty$ for some $a \in \mathbb{C} \backslash\{0,1\}$. (The points $x_{5}=-11 \pm 2 \mathrm{i}$ are not cusps. They are the order-2 elliptic fixed points of $X_{0}(5)$, arising from self-isogenies of lemniscatic elliptic curves; cf. [12, p. 49].) The resulting alternative expression for $h_{5}$ is not pleasing, since it involves the radical $\mathrm{i}=\sqrt{-1}$, and is not given here. 
Proposition 6.1. Let $\mathbb{C}$-valued analytic functions $h_{2}, h_{3}, h_{4}$ be defined in a neighborhood of $0 \in \mathbb{C}$ by (6.1), (6.2), (6.3), in terms of ${ }_{2} F_{1}$. Then for all $x$ near 0 ,

$$
\begin{aligned}
& h_{2}(x(x+16)) \\
& \quad=2[x+16]^{-1 / 4} h_{2}\left(\frac{x^{2}}{x+16}\right), \\
& h_{3}\left(x\left(x^{2}+9 x+27\right)\right) \\
& \quad=3\left[x^{2}+9 x+27\right]^{-1 / 3} h_{3}\left(\frac{x^{3}}{x^{2}+9 x+27}\right), \\
& h_{4}\left(x(x+4)\left(x^{2}+4 x+8\right)\right) \\
& \quad=4\left[(x+2)\left(x^{2}+4 x+8\right)\right]^{-1 / 2} h_{4}\left(\frac{x^{4}}{(x+2)\left(x^{2}+4 x+8\right)}\right) .
\end{aligned}
$$

That is, they respectively satisfy quadratic, cubic, and quartic functional equations.

Proof. These follow from Theorem 5.1 and the appendix. The argument of the left $h_{N}$ is the polynomial expression $R\left(x_{N^{2}}\right)$ for $x_{N}$ in terms of $x_{N^{2}}$, and that of the right $h_{N}$ is the rational expression $x_{N^{2}}^{N} / S^{\prime}\left(x_{N^{2}}\right)$ for $x_{N}^{\prime}$ in terms of $x_{N^{2}}$.

The corresponding identity involving $h_{5}$ appeared above as Proposition 1.1 and will not be repeated here. The three-term recurrence (1.2), which is a simpler characterization of $h_{5}$ than any expression involving ${ }_{2} F_{1}$ or $H l$, is obtained by substituting $h_{5}(z)=\sum_{n=0}^{\infty} c_{n} z^{n}$ into the lifted differential equation (1.4).

Corollary 6.2. The following hypergeometric identities are valid for all $\hat{x}$ in a neighborhood of 0 :

$$
\begin{aligned}
& { }_{2} F_{1}\left(\frac{1}{4}, \frac{3}{4} ; 1 ; 1-\left(\frac{1-\hat{x}}{1+3 \hat{x}}\right)^{2}\right)=(1+3 \hat{x})^{1 / 2}{ }_{2} F_{1}\left(\frac{1}{4}, \frac{3}{4} ; 1 ; \hat{x}^{2}\right), \\
& { }_{2} F_{1}\left(\frac{1}{3}, \frac{2}{3} ; 1 ; 1-\left(\frac{1-\hat{x}}{1+2 \hat{x}}\right)^{3}\right)=(1+2 \hat{x}){ }_{2} F_{1}\left(\frac{1}{3}, \frac{2}{3} ; 1 ; \hat{x}^{3}\right), \\
& { }_{2} F_{1}\left(\frac{1}{2}, \frac{1}{2} ; 1 ; 1-\left(\frac{1-\hat{x}}{1+\hat{x}}\right)^{4}\right)=(1+\hat{x})^{2}{ }_{2} F_{1}\left(\frac{1}{2}, \frac{1}{2} ; 1 ; \hat{x}^{4}\right) .
\end{aligned}
$$

Proof. (i) Express $h_{2}, h_{3}, h_{4}$ above in terms of ${ }_{2} F_{1}$. (ii) Apply Pfaff's transformation ${ }_{2} F_{1}(\alpha, \beta ; \gamma ; s)=(1-s)^{-\alpha}{ }_{2} F_{1}(\alpha, \gamma-\beta ; \gamma ; s /(s-1))$ to each ${ }_{2} F_{1}$. (iii) Rewrite each equation in terms of a new (Möbius-transformed) Hauptmodul $\hat{x}$, defined to equal $x /(x+32), x /(x+9), x /(x+4)$, respectively.

These three transformations of ${ }_{2} F_{1}$ are the quadratic arithmetic-geometric mean (AGM) iteration in signature 4 , the cubic one in signature 3 , and the quartic one in signature 2. The most familiar is the last, which is classical. It is an iterate of Landen's quadratic transformation of the first complete elliptic integral function $\mathrm{K}(\cdot)=\frac{\pi}{2}{ }_{2} F_{1}\left(\frac{1}{2}, \frac{1}{2} ; 1 ; \cdot\right)$. The other two were found by Ramanujan [2, pp. 97, 146]. They play a major role in his theory of elliptic functions to alternative bases.

Our new derivation of these hypergeometric transformations is of interest not only because it clarifies their modular origin, but because it extends to $N=5,6,7$. Proposition 1.1, the $N=5$ transformation, does not fit into Ramanujan's framework. The $N=6,7$ transformations are even more exotic. 
6.2. The $g\left(X_{0}(N)\right)=0, g\left(X_{0}\left(N^{2}\right)\right)>0, g\left(X_{0}^{+}\left(N^{2}\right)\right)=0$ cases $(N=6,7)$. When $N$ equals 6 or 7 , the fundamental weight- 1 modular form $h_{N}$ for $\Gamma_{0}(N)$ can be expressed in terms of ${ }_{2} F_{1}$ on a neighborhood of the point $x_{N}=0$ of $X_{0}(N)$ by

$$
\begin{aligned}
h_{6}(z)= & {\left[\frac{1}{2^{12} 3^{6}}(z+6)^{3}\left(z^{3}+18 z^{2}+84 z+24\right)^{3}\right]^{-1 / 12} } \\
& \quad \times{ }_{2} F_{1}\left(\frac{1}{12}, \frac{5}{12} ; 1 ; \frac{1728 z(z+9)^{2}(z+8)^{3}}{(z+6)^{3}\left(z^{3}+18 z^{2}+84 z+24\right)^{3}}\right) \\
= & H l\left(\frac{9}{8}, \frac{3}{4} ; 1,1,1,1 ;-z / 8\right) ; \\
h_{7}(z)= & {\left[\frac{1}{49}\left(z^{2}+5 z+1\right)^{3}\left(z^{2}+13 z+49\right)\right]^{-1 / 12} } \\
& \quad \times{ }_{2} F_{1}\left(\frac{1}{12}, \frac{5}{12} ; 1 ; \frac{172 z}{\left(z^{2}+5 z+1\right)^{3}\left(z^{2}+13 z+49\right)}\right),
\end{aligned}
$$

where $z$ signifies $x_{N}$. The formulas (6.6a), (6.7a) follow from Definition 4.7, together with the formulas $j=P_{N}\left(x_{N}\right) / Q_{N}\left(x_{N}\right)$ for the covering $\pi_{N}: X_{0}(N) \rightarrow X(1)$ given

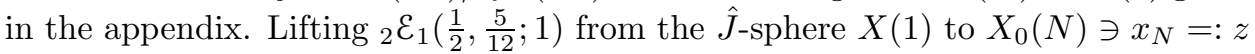
yields an equation based on an operator like (4.2), which if $N=6,7$ is respectively

$$
\begin{aligned}
& \left\{D_{z}^{2}+\left[\frac{1}{z}+\frac{1}{z+8}+\frac{1}{z+9}\right] D_{z}+\left[\frac{z+6}{z(z+8)(z+9)}\right]\right\} h_{6}=0, \\
& \left\{D_{z}^{2}+\left[\frac{1}{z}+\frac{2(2 z+13)}{3\left(z^{2}+13 z+49\right)}\right] D_{z}+\left[\frac{4 z+21}{9 z\left(z^{2}+13 z+49\right)}\right]\right\} h_{7}=0 .
\end{aligned}
$$

The four singular points of (6.8) are $0,-8,-9, \infty$, so a linear rescaling $\hat{z}=-z / 8$ yields the much simpler expression for $h_{6}$ given in (6.6b), in terms of $H l$. The singular points of (6.9) are $0,-\frac{13}{2} \pm \frac{3}{2} \sqrt{-3}, \infty$. (The points $x_{7}=-\frac{13}{2} \pm \frac{3}{2} \sqrt{-3}$ are not cusps. They are the order-3 elliptic fixed points of $X_{0}(7)$, arising from self-isogenies of equianharmonic elliptic curves.) So when $N=7$, just as when $N=5$, the three finite singular points of the lifted equation are not collinear. A reduction of $h_{7}$ to $\mathrm{Hl}$ must accordingly be performed by an awkward Möbius transformation that involves the radical $\sqrt{-3}$. The resulting expression is unpleasing and is omitted.

The $N=6$ case is a bit special, since each of the four singular points of (6.8) on $X_{0}(6)$ is a cusp, there being no elliptic fixed points. That is, $\sigma_{\infty}(6)=4$ and $\varepsilon_{\mathrm{i}}(6)=\varepsilon_{\rho}(6)=0$. It is a result of Beauville [1] and Sebbar 28 that there are only six genus- 0 algebraic curves of the form $\Gamma_{1} \backslash \mathfrak{H}^{*}$, where $\Gamma_{1}<\Gamma$ is a congruence group, with exactly four cusps and no elliptic points. The number of curves shrinks to four if isomorphic curves arising from subgroups $\Gamma_{1}$ that are conjugate in $\operatorname{PSL}(2, \mathbb{R})$ are identified [4, 28. The list includes $X_{0}(6)$, and also $X_{0}(8), X_{0}(9)$, and $X_{1}(5)$.

Proposition 6.3. Let $h_{6}, a \mathbb{C}$-valued function, be defined in a neighborhood of $0 \in \mathbb{C}$ by $h_{6}(z)=\sum_{n=0}^{\infty} c_{n} z^{n}$, where the coefficients satisfy the three-term recurrence

$$
n^{2} c_{n-1}+\left(17 n^{2}+17 n+6\right) c_{n}+72(n+1)^{2} c_{n+1}=0,
$$

initialized by $c_{-1}=0$ and $c_{0}=1$. Equivalently, let $h_{6}$ be defined in terms of ${ }_{2} F_{1}$ or $\mathrm{Hl}$ by (6.6). Let algebraic functions $z=z(t), z^{\prime}=z^{\prime}(t)$ be defined implicitly, in a neighborhood of $t=\infty \in \mathbb{P}^{1}(\mathbb{C})$, by

$$
\begin{aligned}
z+72 / z^{\prime} & =(t-2)\left(t^{5}-10 t^{4}+28 t^{3}-26 t^{2}+20 t+4\right), \\
z \cdot\left[72 / z^{\prime}\right] & =72 t(t-2)^{2}\left(t^{2}-t+1\right) .
\end{aligned}
$$


(Of the two branches, the one on which $z, z^{\prime} \rightarrow 0$ as $t \rightarrow \infty$ is to be chosen.) Then for all $t$ in a neighborhood of $\infty$,

$$
h_{6}(z(t))=6\left\{\frac{z(t)^{6}}{z^{\prime}(t)} \cdot \frac{[z(t)+9]^{3}}{\left[z^{\prime}(t)+9\right]^{2}} \cdot \frac{[z(t)+8]^{2}}{\left[z^{\prime}(t)+8\right]^{3}}\right\}^{-1 / 12} h_{6}\left(z^{\prime}(t)\right)
$$

Remark 6.3.1. By examination, $z \sim 72 / t$ and $z^{\prime} \sim 72 / t^{6}$ as $t \rightarrow \infty$. So (6.12) is a sextic functional equation for $h_{6}$. The multiplier, i.e., the prefactor multiplying $h_{6}$ on the right-hand side, tends to unity as $t \rightarrow \infty$.

Remark 6.3.2. The sequence $d_{n}:=72^{n} c_{n}, n \geq 0$, of Maclaurin coefficients of $h_{6}(72 z)$ is an integral sequence. It begins $1,-6,42,-312,2394,-18756,149136$, $-1199232, \ldots$ A three-term recurrence for $d_{n}$ equivalent to (6.10) was discovered by Coster [11, in an investigation of Beauville's six curves. The representation $d_{n}=\sum_{k=0}^{n}\left(\begin{array}{c}n \\ k\end{array}\right)(-8)^{k} \sum_{j=0}^{n-k}\left(\begin{array}{c}n-k \\ j\end{array}\right)^{3}$ was later worked out by Verrill [33, Table 2], who began with the differential equation satisfied by $h_{6}=[1]^{6}[6] /[2]^{3}[3]^{2}$ as a function of $x_{6} / 72=[2][6]^{5} /[1]^{5}[3]$, i.e., in effect with (6.8). This sequence now appears in Sloane's On-Line Encyclopedia [30] as sequence A093388.

Perhaps due to the simplicity of the Heun representation (6.6b) , the function $h_{6}$ is combinatorially significant. For example, the perimeter generating function for three-dimensional staircase polygons can be expressed in terms of $h_{6}$ [18.

Proof of Proposition 6.3. The recurrence (6.10) comes by substituting $h_{6}(z)=$ $\sum_{n=0}^{\infty} c_{n} z^{n}$ into (6.8). Otherwise, this follows from Theorem 5.3 and the prefactor formula given in Proposition 5.4 with the understanding that $t, z, z^{\prime}$ signify $t_{36}, x_{6}, x_{6}^{\prime}$, the Hauptmoduln of $X_{0}^{+}(36), X_{0}(6), X_{0}(6)^{\prime}$. As the appendix explains, the relation between the sum $x_{6}+72 / x_{6}^{\prime}$ and product $x_{6} \cdot\left[72 / x_{6}^{\prime}\right]$ is parametrized by $t_{36}$. The quantity in curly braces in (6.12) is a product over the $\sigma_{\infty}(6)-1=3$ cusps of $X_{0}(6)$ other than $\left[\frac{1}{1}\right]$. In all, the cusps are $\left[\frac{1}{1}\right] \ni 0,\left[\frac{1}{2}\right],\left[\frac{1}{3}\right],\left[\frac{1}{6}\right] \ni \mathrm{i} \infty$, with respective widths (i.e., multiplicities over $X(1)$ ) equal to $e_{1,6}=6, e_{2,6}=3, e_{3,6}=2$, and $e_{6,6}=1$. A comparison with the formula (A.3) for the covering $j=j\left(x_{6}\right)$ reveals that these cusps are located respectively at the points $x_{6}=\infty,-8,-9,0$ on $X_{0}(6)$. The prefactor follows.

However, Theorem 5.3 does not quite imply that the functions of $t:=t_{36}$ on the two sides of (6.12) are equal. What it implies is that they are the unique (normalized) analytic solutions near the point $\left(t_{36}, s_{36}\right)=(\infty,-\infty)$ on the elliptic curve $X_{0}(36)$ modeled by (A.6), i.e., by

$$
s_{36}^{2}=t_{36}^{4}-8 t_{36}^{3}+12 t_{36}^{2}-8 t_{36}+4
$$

of a pair of differential equations that are the same up to one degree of freedom: their single affine accessory parameter. To verify (6.12), one must show the two equations are identical. They are obtained from (6.8), the equation on $X_{0}(6) \ni x_{6}$ satisfied by $h_{6}$, by (i) lifting along $\left(t_{36}, s_{36}\right) \mapsto x_{6}$, and (ii) lifting along $\left(t_{36}, s_{36}\right) \mapsto x_{6}^{\prime}$ and including the prefactor. Each lifted equation on $X_{0}(36) \ni\left(t_{36}, s_{36}\right)=:(t, s)$ is based on a Fuchsian operator of the normal form (4.3), with $g=1$ and $n=$ $\sigma_{\infty}(36)=12$ singular points (i.e., cusps). Fortunately, the two turn out to be the 
same. Regardless of which route is taken, the lifting of (6.8) to $X_{0}(36)$ is

$$
\begin{aligned}
& \left\{\left(s \frac{d}{d t}\right)^{2}+\left[\frac{s}{t}+\frac{s}{t-1}+\frac{s}{t-2}+\frac{s(2 t-1)}{t^{2}-t+1}+6 t+-6\right]\right]\left(s \frac{d}{d t}\right) \\
& \left.+3\left[\frac{-(s+2)}{t}+\frac{s-6}{t-2}+\frac{-(t+1) s+3 t}{t^{2}-t+1}+\left(6 t^{2}-23 t+8\right)+6 s\right]\right\} \mathfrak{h}_{36}=0 .
\end{aligned}
$$

Here the single affine accessory parameter is boxed, and the $n-3+3 g=12$ projective accessory parameters are contained in the second bracketed expression.

This equation on the equianharmonic elliptic curve $X_{0}(36)$, every ratio of solutions of which is of the form $(a \tau+b) /(c \tau+d)$, is of independent interest. It is a uniformizing differential equation of the sort guaranteed to exist by Theorem 4.2, but few such equations in the case of positive genus have appeared in the literature. By examination, it displays the 12 cusps of $X_{0}(36)$ explicitly. They come in pairs, each pair being related by the Fricke involution $s_{36} \mapsto-s_{36}$, and are located at $t_{36}=0,1,2, \frac{1}{2} \pm \frac{\sqrt{-3}}{2}, \infty$. The 6 rational cusps $\left[\frac{a}{d}\right]_{36}$ are those with $t_{36}=0,1, \infty$. They are singled out by $\varphi((d, N / d))=1$, a standard arithmetic rationality condition [24, and are respectively $\left[\frac{1}{2}\right],\left[\frac{1}{18}\right] ;\left[\frac{1}{4}\right],\left[\frac{1}{9}\right] ;\left[\frac{1}{1}\right] \ni 0,\left[\frac{1}{36}\right] \ni$ i $\infty$.

For present purposes, all that matters is that the value of the single (boxed) affine accessory parameter is independent of which of the two liftings is used. It is actually possible to verify this without deriving (6.14) in full, by simply working out the first two terms in an expansion of its coefficient of $s d / d t$ about $(t, s)=(\infty,-\infty)$, i.e., about $\tau=\mathrm{i} \infty$. This can readily be done by hand, though why the result is the same for the lifting from $X_{0}(6)$ to $X_{0}(36)$ and the weak lifting from $X_{0}(6)^{\prime}$ to $X_{0}(36)$ is not entirely clear.

Proposition 6.4. Let $h_{7}$, a $\mathbb{C}$-valued function, be defined in a neighborhood of $0 \in \mathbb{C}$ by $h_{7}(z)=\sum_{n=0}^{\infty} c_{n} z^{n}$, where the coefficients satisfy the three-term recurrence

$$
(3 n-1)^{2} c_{n-1}+3\left(39 n^{2}+26 n+7\right) c_{n}+441(n+1)^{2} c_{n+1}=0,
$$

initialized by $c_{-1}=0$ and $c_{0}=1$. Equivalently, let $h_{7}$ be defined in terms of ${ }_{2} F_{1}$ by 6.7a). Let algebraic functions $z=z(t), z^{\prime}=z^{\prime}(t)$ be defined implicitly, in a neighborhood of $t=\infty \in \mathbb{P}^{1}(\mathbb{C})$, by

$$
\begin{aligned}
z+49 / z^{\prime} & =\left(t^{3}-7 t^{2}+14 t-7\right)\left(t^{4}-14 t^{3}+63 t^{2}-98 t+35\right) \\
z \cdot\left[49 / z^{\prime}\right] & =49\left(t^{3}-7 t^{2}+14 t-7\right)^{2}
\end{aligned}
$$

(Of the two branches, the one on which $z, z^{\prime} \rightarrow 0$ as $t \rightarrow \infty$ is to be chosen.) Then for all $t$ in a neighborhood of $\infty$,

$$
h_{7}(z(t))=7\left[\frac{z(t)^{7}}{z^{\prime}(t)}\right]^{-1 / 12} h_{7}\left(z^{\prime}(t)\right) .
$$

Remark 6.4.1. By examination, $z \sim 49 / t$ and $z^{\prime} \sim 49 / t^{7}$ as $t \rightarrow \infty$. So (6.17) is a septic functional equation for $h_{7}$. The multiplier tends to unity as $t \rightarrow \infty$.

Remark 6.4.2. The sequence $d_{n}:=441^{n} c_{n}, n \geq 0$, of Maclaurin coefficients of $h_{7}(441 z)$ is an integral sequence. It begins $1,-21,693,-23940,734643,-13697019$, -494620749, 83079255420, .... 
Proof of Proposition 6.4. This resembles the proof of Proposition 6.3 but is simpler because 7 is not composite. Here $t, z, z^{\prime}$ are $t_{49}, x_{7}, x_{7}^{\prime}$, the Hauptmoduln of $X_{0}^{+}(49), X_{0}(7), X_{0}(7)^{\prime}$. There are only two cusps on $X_{0}(7)$, namely $\left[\frac{1}{1}\right] \ni 0$, $\left[\frac{1}{7}\right] \ni \mathrm{i} \infty$, with respective widths $e_{1,7}=7$ and $e_{7,7}=1$. They are located at $x_{7}=\infty, 0$, respectively. So the product (5.2) comprises only a single factor, namely $x_{7}^{7} / x_{7}^{\prime}$, i.e., $z(t)^{7} / z^{\prime}(t)$. As in the $N=6$ case, the lifting of the differential equation (6.9) from $X_{0}(7)$ to the elliptic curve $X_{0}(49)$ and its weak lifting from $X_{0}(7)^{\prime}$ to $X_{0}(49)$ turn out to be identical due to the values of their affine accessory parameters being the same. So the two sides of (6.17) are equal, as claimed.

The cases $N=8,9$ can in principle also be handled by Theorem[5.3, like $N=6,7$, since equations for the curves $X_{0}(64), X_{0}(81)$ are known $\left[29\right.$. $\left(X_{0}(64), X_{0}(81)\right.$ are non-hyperelliptic of genera 3,4 , and $X_{0}(64)$ is the degree- 4 Fermat curve.) The covering maps $\phi_{8}, \phi_{8}^{\prime}, \phi_{9}, \phi_{9}^{\prime}$ could also be worked out. But for both $N=8$ and 9 , a simple parametrization of the relation between $x_{N}$ and $x_{N}^{\prime}$ may be lacking, since $X_{0}^{+}(64), X_{0}^{+}(81)$ are of genus 1 , not 0 .

\section{Possible extensions}

We conclude by mentioning some possible generalizations of our approach. Each identity derived above arises from the covering $\phi_{N}: X_{0}\left(N^{2}\right) \rightarrow X_{0}(N)$ induced by a subgroup relation $\Gamma_{0}(N)>\Gamma_{0}\left(N^{2}\right)$. Under the Fricke involution $w_{N^{2}}, \Gamma_{0}(N)$ is conjugated to $\Gamma_{0}(N)^{\prime}:=w_{N^{2}}^{-1} \Gamma_{0}(N) w_{N^{2}}>\Gamma_{0}\left(N^{2}\right)$, and $\phi_{N}^{\prime}: X_{0}\left(N^{2}\right) \rightarrow X_{0}(N)^{\prime}$ arises from the latter relation. As a first generalization, one could begin instead with the covering $\phi_{N, M}: X_{0}(M N) \rightarrow X_{0}(N)$ induced by $\Gamma_{0}(N)>\Gamma_{0}(M N)$, where $M \neq N$ is allowed. The canonical Hauptmoduln of $X_{0}(M N), X_{0}(N)$ are $x_{M N}, x_{N}$, and that of $w_{M N}^{-1} \Gamma_{0}(N) w_{M N}>\Gamma_{0}(M N)$ is $x_{N}^{\prime \prime}=x_{N}^{\prime \prime}(\tau):=x_{N}(M \tau)$. If for example $N=2$ and $M=3$, this approach turns out to yield the functional equation

$$
h_{2}\left(\frac{x(x+8)^{3}}{x+9}\right)=3[x+9]^{-1 / 2} h_{2}\left(\frac{x^{3}(x+8)}{(x+9)^{3}}\right) \text {, }
$$

where $x:=x_{6}$. The argument of the left $h_{2}$ is the expression (A.4a) for $x_{2}$ in terms of $x_{6}$, and that of the right is $x_{2}^{\prime \prime}=x_{2}(3 \cdot)$, meaning $x_{2}^{\prime \prime}(\tau)=x_{2}(3 \tau)$, in terms of $x_{6}$. This can be rewritten as

$$
\begin{aligned}
{ }_{2} F_{1}\left(\frac{1}{4}, \frac{3}{4} ; 1 ;\right. & \left.\frac{64 p}{\left(3+6 p-p^{2}\right)^{2}}\right) \\
& =\left(\frac{9\left(3+6 p-p^{2}\right)}{27-18 p-p^{2}}\right)^{1 / 2}{ }_{2} F_{1}\left(\frac{1}{4}, \frac{3}{4} ; 1 ; \frac{64 p^{3}}{\left(27-18 p-p^{2}\right)^{2}}\right),
\end{aligned}
$$

a hypergeometric identity valid near $p=0$. This is a known identity: the cubic modular equation in Ramanujan's theory of elliptic functions in signature 4, which has been proved by other means [2, pp. 152-153]. The parameter $p$ is the Möbiustransformed Hauptmodul $x_{6} /\left(x_{6}+8\right)=9[1]^{4}[6]^{8} /[2]^{8}[3]^{4}$ of $X_{0}(6)$, which has a zero at the cusp $\left[\frac{1}{6}\right]_{6} \ni \mathrm{i} \infty$ and a pole at the cusp $\left[\frac{1}{2}\right]_{6}$. A second noteworthy functional equation comes from the choices $N=5$ and $M=2$. It is

$$
h_{5}\left(\frac{x(x+5)^{2}}{x+4}\right)=2[x+4]^{-1 / 2} h_{5}\left(\frac{x^{2}(x+5)}{(x+4)^{2}}\right),
$$

where $x:=x_{10}$. The argument of the left $h_{5}$ is the expression for $x_{5}$ in terms of $x_{10}$, and that of the right is $x_{5}^{\prime \prime}=x_{5}(2 \cdot)$ in terms of $x_{10}$, both expressions being due to 
Fricke. Here $x_{10}$ is the canonical Hauptmodul $20[2][10]^{3} /[1]^{3}[5]$ of $X_{0}(10)$. The identity (7.3) merits comparison with Proposition 1.1.

A further generalization comes from focusing on $h_{N}$, originally given in terms of ${ }_{2} F_{1}$, as a weight- 1 modular form for $\Gamma_{0}(N)$ that necessarily satisfies a secondorder differential equation on $X_{0}(N)$. As an abstraction of this situation, let $f$ be any weight-1 modular form for a congruence subgroup $G$ of genus zero, with Hauptmodul $x_{G}:=x_{G}(\tau)$. Any $g \in S L(2, \mathbb{R})$ will yield a projective action on $\mathfrak{H} \ni \tau$ and a weight-1 modular form $f^{\prime}:=f\left(x_{G}(g(\tau))\right)$ for the conjugated group $G^{\prime}:=$ $g^{-1} G g$, which has Hauptmodul $x_{G^{\prime}}=x_{G^{\prime}}(\tau):=x_{G}(g(\tau))$. So both $f$ and $f^{\prime}$ will be weight-1 modular forms for $\tilde{G}:=G \cap G^{\prime}$. The simplest case is when $\tilde{G}$ is of genus zero as well, with a Hauptmodul $x_{\tilde{G}}$. In this case $x_{G}, x_{G^{\prime}}$, and the weight-zero form $f^{\prime} / f$ will all be expressible in terms of $x_{\tilde{G}}$, yielding an identity of the form

$$
f\left(x_{G}\left(x_{\tilde{G}}\right)\right)=A\left(x_{\tilde{G}}\right) f\left(x_{G^{\prime}}\left(x_{\tilde{G}}\right)\right),
$$

with $A(\cdot)$ a fractional power of some rational function. The functional equations derived above for $h_{N}, N=2, \ldots, 5$, were all of this type.

In general the identity (7.4) will have no interpretation as a transformation of ${ }_{2} F_{1}$, since the differential equation satisfied by $f$ will not be of hypergeometric type (though it will be of second order). However, an additional extension suggests itself. If $f$ has weight $k, k \geq 1$, the differential equation will be of order $k+1$. For example, it could be the order- $(k+1)$ differential equation satisfied by any generalized hypergeometric function of the type ${ }_{k+1} F_{k}$. The possibility therefore exists that by an approach similar to the one used in this article, one could derive functional equations satisfied by certain ${ }_{k+1} F_{k}, k>1$. This remains to be explored.

\section{Appendix. Hauptmoduln}

If $X_{0}(N)$ is of genus 0 , then it has a Hauptmodul $x_{N}$, which may be regarded as a function on $\mathfrak{H} \ni \tau$. This Hauptmodul is determined uniquely by the condition that it have divisor $(\mathrm{i} \infty)-(0)$, i.e., a simple zero (resp. pole) at the cusp $\tau=\mathrm{i} \infty$ (resp. the cusp $\tau=0$ ), together with a standard normalization condition that $x_{N}(-1 / N \tau)$ have a Fourier expansion on $\mathfrak{H}$ that begins $q^{-1}+\cdots$, where $q:=e^{2 \pi \mathrm{i} \tau}$. The $j$-invariant will be a rational function of $x_{N}$, and $x_{N}$ itself will be in the function field of $X_{0}\left(N^{2}\right)$, and in fact of $X_{0}(M N)$ for every $M \geq 1$.

This appendix collects the formulas of the form $j=j\left(x_{N}\right)$, and for $x_{N}$ itself, which are used in the body of the article. N. Fine's notation $[k]$ for the function $\eta(k \cdot)$, i.e., for $\eta(k \tau), \tau \in \mathfrak{H}$, is used to save space. Here $\eta$ is the Dedekind eta function $q^{1 / 24} \prod_{n \in \mathbb{N}}\left(1-q^{n}\right)$, satisfying $\eta(\tau+1)=e^{\pi \mathrm{i} / 12} \eta(\tau), \eta(-1 / \tau)=(-\mathrm{i} \tau)^{1 / 2} \eta(\tau)$.

The simplest cases are $N=2,3,4,5$, when $X_{0}\left(N^{2}\right)$ like $X_{0}(N)$ is of genus 0 , and a Hauptmodul $x_{N^{2}}$ exists.

- $N=2 . \quad x_{2}:=2^{12}[2]^{24} /[1]^{24}$, and $x_{2}$ satisfies $x_{2}(\tau) x_{2}(-1 / 2 \tau)=2^{12}$. The equality $j=\left(x_{2}+16\right)^{3} / x_{2}$ holds. $x_{4}:=2^{8}[4]^{8} /[1]^{8}$ is the Hauptmodul on $X_{0}(4)$, and $x_{2}=x_{4}\left(x_{4}+16\right)$. The Hauptmodul $x_{4}$ satisfies $x_{4}(\tau) x_{4}(-1 / 4 \tau)=2^{8}$.

- $N=3 . x_{3}:=3^{6}[3]^{12} /[1]^{12}$, and $x_{3}$ satisfies $x_{3}(\tau) x_{3}(-1 / 3 \tau)=3^{6}$. The equality $j=\left(x_{3}+3\right)^{3}\left(x_{3}+27\right) / x_{3}$ holds. $x_{9}:=3^{3}[9]^{3} /[1]^{3}$ is the Hauptmodul on $X_{0}(9)$, and $x_{3}=x_{9}\left(x_{9}^{2}+9 x_{9}+27\right)$. The Hauptmodul $x_{9}$ satisfies $x_{9}(\tau) x_{9}(-1 / 9 \tau)=3^{3}$. 
- $N=4 . \quad x_{4}:=2^{8}[4]^{8} /[1]^{8}$, and $x_{4}$ satisfies $x_{4}(\tau) x_{4}(-1 / 4 \tau)=2^{8}$. The equality $j=\left(x_{4}^{2}+16 x_{4}+16\right)^{3} /\left(x_{4}\left(x_{4}+16\right)\right)$ holds. $x_{16}:=2^{3}[2][16]^{2} /[1]^{2}[8]$ is the Hauptmodul on $X_{0}(16)$, and $x_{4}=x_{16}\left(x_{16}+4\right)\left(x_{16}^{2}+4 x_{16}+8\right)$. The Hauptmodul $x_{16}$ satisfies $x_{16}(\tau) x_{16}(-1 / 16 \tau)=2^{3}$.

- $N=5 . \quad x_{5}:=5^{3}[5]^{6} /[1]^{6}$, and $x_{5}$ satisfies $x_{5}(\tau) x_{5}(-1 / 5 \tau)=5^{3}$. The equality $j=\left(x_{5}^{2}+10 x_{5}+5\right)^{3} / x_{5}$ holds. $x_{25}:=5[25] /[1]$ is the Hauptmodul on $X_{0}(25)$, and $x_{5}=x_{25}\left(x_{25}^{4}+5 x_{25}^{3}+15 x_{25}^{2}+25 x_{25}+25\right)$. The Hauptmodul $x_{25}$ satisfies $x_{25}(\tau) x_{25}(-1 / 25 \tau)=5$.

Of the preceding formulas, the most familiar are the definition of $x_{N}$ as an eta product and the expression for $j$ in terms of $x_{N}$, when $N=2,3,5$. Like the corresponding formulas for $N=7,13$, they go back to Weber, indeed to Klein, and are often reproduced [12, $\S 4$ ]. The remaining formulas were extracted from Fricke [15, II. Abschnitt, 4. Kap.], with due care. Fricke occasionally varied the normalization of his Hauptmoduln $\tau_{N}$, corresponding to $x_{N}$, and his $\tau_{2}(\cdot)$ is proportional to $x_{2}(2 \cdot)$ rather than to $x_{2}(\cdot)$. He also did not give eta-product expressions for his $\tau_{4}, \tau_{16}$, but they are easily worked out [23. Knopp [21, § 7.6] gives an independent derivation of the quintic formula for $x_{5}$ in terms of $x_{25}$.

From each expression for $j$ in terms of $x_{N}$, one obtains an expression for $j_{N}$, where $j_{N}(\tau):=j(N \tau)$, by applying the involution $\tau \mapsto-1 / \tau$ to both sides. Thus, $j_{2}=\left(x_{2}+256\right)^{3} / x_{2}^{2}$. Similarly, applying the Fricke involution $w_{N^{2}}: \tau \mapsto-1 / N^{2} \tau$ to both sides of the polynomial expression for $x_{N}$ in terms of $x_{N^{2}}$ yields a nonpolynomial rational expression for $x_{N}^{\prime}$, where $x_{N}^{\prime}(\tau):=x_{N}(N \tau)$. For example, $x_{2}^{\prime}=x_{4}^{2} /\left(x_{4}+16\right)$; also $x_{3}^{\prime}=x_{9}^{3} /\left(x_{9}^{2}+9 x_{9}+27\right)$ and $x_{4}^{\prime}=x_{16}^{4} /\left(x_{16}+2\right)\left(x_{16}^{2}+4 x_{16}+8\right)$. In the same way, $x_{5}^{\prime}=x_{25}^{5} /\left(x_{25}^{4}+5 x_{25}^{3}+15 x_{25}^{2}+25 x_{25}+25\right)$.

If $N=6,7$, then $X_{0}\left(N^{2}\right)$ is of genus 1 , but $X_{0}^{+}\left(N^{2}\right)=X_{0}\left(N^{2}\right) /\left\langle w_{N^{2}}\right\rangle$ is of genus 0 and has a Hauptmodul $t_{N^{2}}$ that lifts to a double-valued function on $X_{0}\left(N^{2}\right)$. The following is a reformulation of Fricke's treatment of this situation, along the lines of Cohn [9]. A general point on $X_{0}\left(N^{2}\right)$ is determined by the pair $\left(t_{N^{2}}, s_{N^{2}}\right)$, where

$$
s_{N^{2}}^{2}=\mathrm{P}_{N^{2}}\left(t_{N^{2}}\right),
$$

with $\mathrm{P}_{N^{2}}$ a polynomial of degree 4 over $\mathbb{C}$ (with simple roots). The projective curve corresponding to this affine quartic has two infinite points: informally, $\left(t_{N^{2}}, s_{N^{2}}\right)=$ $(\infty,-\infty),(\infty,+\infty)$. In Fricke's normalization convention, these are taken to be the distinguished cusps $\tau=\mathrm{i} \infty, 0$, and $t_{N^{2}}$ is normalized so that the invariants $j, j_{N^{2}}$ are asymptotic to $t_{N^{2}}, t_{N^{2}}^{N^{2}}$ and $t_{N^{2}}^{N^{2}}, t_{N^{2}}$ as $t_{N^{2}} \rightarrow \infty$ along the branches leading to $\tau=\mathrm{i} \infty$ and $\tau=0$ respectively [9, (1.11)]. Also, $\mathrm{P}_{N^{2}}$ is taken to be monic. It then mysteriously turns out that $\mathrm{P}_{N^{2}}$ is in $\mathbb{Z}\left[t_{N^{2}}\right]$; this may be fortuitous [10, $\left.\S 1\right]$.

It will be the case that $x_{N}(\tau) x_{N}(-1 / N \tau)=\kappa_{N}$ for some $\kappa_{N} \in \mathbb{N}$. So, applying the involution $w_{N^{2}}: \tau \mapsto-1 / N^{2} \tau$ to the function $x_{N}(\tau)$ yields $\kappa_{N} / x_{N}(N \tau)$. It follows that a rational function $F_{N}\left(t_{N^{2}}, s_{N^{2}}\right)$ exists on $X_{0}\left(N^{2}\right)$ such that

$$
\begin{aligned}
x_{N}(\tau) & =F_{N}\left(t_{N^{2}}, s_{N^{2}}\right), \\
\kappa_{N} / x_{N}(N \tau) & =F_{N}\left(t_{N^{2}},-s_{N^{2}}\right) .
\end{aligned}
$$

Any such function is linear in $s_{N^{2}}$. Consequently, the sum and product of $x_{N}(\tau)$, $\kappa_{N} / x_{N}(N \tau)$ are each rational functions of $t_{N^{2}}$ alone. That is, the relation between the sum and product is of genus 0: it may be uniformized by rational functions. 
- $N=6 . x_{6}:=72[2][6]^{5} /[1]^{5}[3]$, and $x_{6}$ satisfies $x_{6}(\tau) x_{6}(-1 / 6 \tau)=72$. The 12 -sheeted cover of $X(1) \cong \mathbb{P}^{1}(\mathbb{C})_{j}$ by $X_{0}(6) \cong \mathbb{P}^{1}(\mathbb{C})_{x_{6}}$ is given by

$$
j=\frac{\left(x_{6}+6\right)^{3}\left(x_{6}^{3}+18 x_{6}^{2}+84 x_{6}+24\right)^{3}}{x_{6}\left(x_{6}+9\right)^{2}\left(x_{6}+8\right)^{3}} .
$$

As elements of the function field of $X_{0}(6)$, the functions $x_{2}, x_{3}$ may be expressed rationally as

$$
\begin{aligned}
& x_{2}=\frac{x_{6}\left(x_{6}+8\right)^{3}}{x_{6}+9}, \\
& x_{3}=\frac{x_{6}\left(x_{6}+9\right)^{2}}{x_{6}+8} .
\end{aligned}
$$

Combined with the eta-product formulas for $x_{2}, x_{3}, x_{6}$, these imply

$$
\begin{aligned}
& x_{6}+8=8[2]^{9}[3]^{3} /[1]^{9}[6]^{3}, \\
& x_{6}+9=9[2]^{4}[3]^{8} /[1]^{8}[6]^{4} .
\end{aligned}
$$

The double cover of $X_{0}^{+}(36) \ni t_{36}$ by $X_{0}(36) \ni\left(t_{36}, s_{36}\right)$ is given by

$$
s_{36}^{2}=t_{36}^{4}-8 t_{36}^{3}+12 t_{36}^{2}-8 t_{36}+4,
$$

from which it follows that as an elliptic curve, $X_{0}(36)$ is equianharmonic: it has $j$-invariant zero. It is a 6 -sheeted cover of $X_{0}(6)$. As elements of the function field of $X_{0}(36)$, the functions $x_{6}(\tau), 72 / x_{6}(6 \tau)$ may be written as

(A.7) $\frac{1}{2}\left(t_{36}-2\right)$

$\times\left[\left(t_{36}^{5}-10 t_{36}^{4}+28 t_{36}^{3}-26 t_{36}^{2}+20 t_{36}+4\right) \pm\left(t_{36}^{3}-6 t_{36}^{2}+6 t_{36}-2\right) s_{36}\right]$.

The formulas

$$
\begin{aligned}
x_{6}(\tau)+72 / x_{6}(6 \tau)= & \left(t_{36}-2\right) \\
& \times\left(t_{36}^{5}-10 t_{36}^{4}+28 t_{36}^{3}-26 t_{36}^{2}+20 t_{36}+4\right), \\
x_{6}(\tau) \cdot\left[72 / x_{6}(6 \tau)\right]= & 72 t_{36}\left(t_{36}-2\right)^{2}\left(t_{36}^{2}-t_{36}+1\right)
\end{aligned}
$$

uniformize the sum-product relation.

- $N=7 . x_{7}:=49[7]^{4} /[1]^{4}$, and $x_{7}$ satisfies $x_{7}(\tau) x_{7}(-1 / 7 \tau)=49$. The 8-sheeted cover of $X(1) \cong \mathbb{P}^{1}(\mathbb{C})_{j}$ by $X_{0}(7) \cong \mathbb{P}^{1}(\mathbb{C})_{x_{7}}$ is given by

$$
j=\frac{\left(x_{7}^{2}+5 x_{7}+1\right)^{3}\left(x_{7}^{2}+13 x_{7}+49\right)}{x_{7}} .
$$

The double cover of $X_{0}^{+}(49) \ni t_{49}$ by $X_{0}(49) \ni\left(t_{49}, s_{49}\right)$ is given by

$$
s_{49}^{2}=t_{49}^{4}-14 t_{49}^{3}+63 t_{49}^{2}-98 t_{49}+21,
$$

from which it follows that, as an elliptic curve, $X_{0}(49)$ has $j$-invariant $-15^{3}$. It is a 7-sheeted cover of $X_{0}(7)$. As elements of the function field of $X_{0}(49)$, the functions $x_{7}(\tau), 49 / x_{7}(7 \tau)$ may be written as

$$
\begin{aligned}
\frac{1}{2}\left(t_{49}^{3}\right. & \left.-7 t_{49}^{2}+14 t_{49}-7\right) \\
& \times\left[\left(t_{49}^{4}-14 t_{49}^{3}+63 t_{49}^{2}-98 t_{49}+35\right) \pm\left(t_{49}^{2}-7 t_{49}+7\right) s_{49}\right] .
\end{aligned}
$$


The formulas

$$
\begin{aligned}
x_{7}(\tau)+49 / x_{7}(7 \tau)= & \left(t_{49}^{3}-7 t_{49}^{2}+14 t_{49}-7\right) \\
& \times\left(t_{49}^{4}-14 t_{49}^{3}+63 t_{49}^{2}-98 t_{49}+35\right), \\
x_{7}(\tau) \cdot\left[49 / x_{7}(7 \tau)\right]= & 49\left(t_{49}^{3}-7 t_{49}^{2}+14 t_{49}-7\right)^{2}
\end{aligned}
$$

uniformize the sum-product relation.

The above formulas for the $N=6,7$ cases follow from those given by Fricke, with a good bit of massaging. For instance, he constructs $X_{0}(36)$ as a double cover of the genus- 0 curve $X_{0}(18)$ and works out not a formula for $x_{6}$ in terms of $\left(t_{36}, s_{36}\right)$, but rather a formula for the Hauptmodul $x_{18}:=6[2][3][18]^{2} /[1]^{2}[6][9]$ of $X_{0}(18)$. However, $x_{6}=x_{18}\left(x_{18}^{2}+6 x_{18}+12\right)$, as he computes elsewhere.

It can be shown that $t_{36}-1=[4][9] /[1][36]$, relating his quartic equation (A.6) to the equivalent equation for $X_{0}(36)$ derived by González Rovira [16, §4.3]. Two other notable identities involving $t_{36}$ were obtained by Kluit [20], namely

$$
\begin{aligned}
& t_{36}=[2]^{3}[3][12][18]^{3} /[1]^{2}[4][6]^{2}[9][36]^{2}, \\
& t_{36}-2=[4][6]^{8}[9] /[2]^{2}[3]^{3}[12]^{3}[18]^{2} .
\end{aligned}
$$

(These eta-products also appear in the tabulation of Ford et al. [13, Table 4]; see line 36D.) The further eta-function identity

$$
t_{36}^{2}-t_{36}+1=[2]^{2}[3]^{4}[12]^{4}[18]^{2} /[1]^{3}[4][6]^{4}[9][36]^{3}
$$

follows by substituting (A.13 b) into the right side of (A.8b). Level 36 is in fact rich in relations among eta products. For identities relating eta products that are weight-1 modular forms on the quotient curve $X_{0}^{+}(36)$, see Köhler [22].

\section{ACKNOWLEDGEMENTS}

The author would like to thank the referee for valuable comments and suggestions, which strengthened this article.

\section{REFERENCES}

[1] A. Beauville, Les familles stables de courbes elliptiques sur $\mathbf{P}^{1}$ admettant quatre fibres singulières, C. R. Acad. Sci. Paris Sér. I Math. 294 (1982), no. 19, 657-660. MR0664643 (83h:14008)

[2] B. C. Berndt, Ramanujan's Notebooks, Part V, Springer-Verlag, New York/Berlin, 1998. MR.1486573 (99f:11024)

[3] B. C. Berndt, S. Bhargava, and F. G. Garvan, Ramanujan's theories of elliptic functions to alternative bases, Trans. Amer. Math. Soc. 347 (1995), no. 11, 4163-4244. MR1311903 (97h:33034)

[4] F. Beukers, On Dwork's accessory parameter problem, Math. Z. 241 (2002), no. 2, 425-444. MR 1935494 (2003i:12013)

[5] J. M. Borwein and P. B. Borwein, A cubic counterpart of Jacobi's identity and the AGM, Trans. Amer. Math. Soc. 323 (1991), no. 2, 691-701. MR1010408(91e:33012)

[6] J. M. Borwein, P. B. Borwein, and F. G. Garvan, Some cubic modular identities of Ramanujan, Trans. Amer. Math. Soc. 343 (1994), no. 1, 35-47. MR1243610 (94j:11019)

[7] H. H. Chan, On Ramanujan's cubic transformation formula for ${ }_{2} F_{1}\left(\frac{1}{3}, \frac{2}{3} ; 1 ; z\right)$, Math. Proc. Cambridge Philos. Soc. 124 (1998), no. 2, 193-204. MR.1631107 (99f:11054)

[8] K. Chandrasekharan, Elliptic Functions, Die Grundlehren der mathematischen Wissenschaften, no. 281, Springer-Verlag, New York/Berlin, 1985. MR0808396 (87e:11058)

[9] H. Cohn, Fricke's two-valued modular equations, Math. Comp. 51 (1988), no. 184, 787-807. MR0935079 (89f:11064) 
[10] Computation of singular moduli by multi-valued modular equations, Computational Number Theory (A. Pethő, M. E. Pohst, H. C. Williams, and H. G. Zimmer, eds.), Walter de Gruyter, Berlin, 1991, pp. 213-225. MR1151866 (93e:11059)

[11] M. J. Coster, Over 6 families van krommen [On 6 families of curves], Master's thesis, Rijksuniversiteit Leiden, Leiden, The Netherlands, August 1983.

[12] N. D. Elkies, Elliptic and modular curves over finite fields and related computational issues, Computational Perspectives in Number Theory (D. A. Buell and J. T. Teitelbaum, eds.), AMS/IP Studies in Advanced Mathematics, no. 7, American Mathematical Society, Providence, RI, 1998, pp. 21-76. MR1486831 (99a:11078)

[13] D. Ford, J. McKay, and S. Norton, More on replicable functions, Comm. Algebra 22 (1994), no. 13, 5175-5193. MR1291027 (95i:11036)

[14] L. R. Ford, Automorphic Functions, 2nd ed., Chelsea Publishing Co., New York, 1951.

[15] R. Fricke, Die algebraischen Ausführungen, Die elliptischen Funktionen und ihre Anwendungen, vol. II, B. G. Teubner, Leipzig, Germany, 1922.

[16] J. González Rovira, Equations of hyperelliptic modular curves, Ann. Inst. Fourier (Grenoble) 41 (1991), no. 4, 779-795. MR1150566 (93g:11064)

[17] É. Goursat, Sur l'équation différentielle linéaire qui admet pour intégrale la série hypergéométrique, Ann. Sci. École Normale Sup. (2) 10 (1881), S3-S142. MR1508709

[18] A. J. Guttmann and T. Prellberg, Staircase polygons, elliptic integrals, Heun functions, and lattice Green functions, Phys. Rev. E 47 (1993), no. 4, R2233-R2236.

[19] J. Harnad and J. McKay, Modular solutions to equations of generalized Halphen type, Proc. Roy. Soc. London Ser. A 456 (2000), no. 1994, 261-294. MR1811320 (2002g:11047)

[20] P. G. Kluit, On the normalizer of $\Gamma_{0}(N)$, Modular Functions of One Variable, V (J.-P. Serre and D. Zagier, eds.), Lecture Notes in Mathematics, no. 601, Springer-Verlag, New York/Berlin, 1977, pp. 239-246. MR0480340 (58:513)

[21] M. I. Knopp, Modular Functions in Analytic Number Theory, Markham Publishing Co., Chicago, IL, 1970. MR0265287 (42:198)

[22] G. Köhler, Eta products of weight 1 and level 36, Arch. Math. (Basel) 76 (2001), no. 3, 202-214. MR.1816991 (2001k:11070)

[23] M. Newman, Construction and application of a class of modular functions (II), Proc. London Math. Soc. 9 (1958), no. 35, 373-387. MR0107629(21:6354)

[24] A. P. Ogg, Rational points on certain elliptic modular curves, Analytic Number Theory (H. G. Diamond, ed.), Proceedings of Symposia in Pure Mathematics, vol. XXIV, American Mathematical Society, Providence, RI, 1973, pp. 221-231. MR0337974 (49:2743)

[25] E. G. C. Poole, Introduction to the Theory of Linear Differential Equations, Oxford University Press, Oxford, 1936. Dover reprint, 1960. MR.0111886 (22:2746)

[26] A. Ronveaux (ed.), Heun's Differential Equations, Oxford University Press, Oxford, 1995. MR 1392976 (98a:33005)

[27] B. Schoeneberg, Elliptic Modular Functions, Die Grundlehren der mathematischen Wissenschaften, no. 203, Springer-Verlag, New York/Berlin, 1974. MR0412107 (54:236)

[28] A. Sebbar, Modular subgroups, forms, curves and surfaces, Canad. Math. Bull. 45 (2002), no. 2, 294-308. MR.1904094 (2003d:20064)

[29] M. Shimura, Defining equations of modular curves $X_{0}(N)$, Tokyo J. Math. 18 (1995), no. 2, 443-456. MR1363479 (96j:11085)

[30] N. J. A. Sloane, The On-Line Encyclopedia of Integer Sequences, currently available at http://www.research.att.com/ $n$ njas/sequences/, 2005.

[31] P. F. Stiller, Special values of Dirichlet Series, Monodromy, and the Periods of Automorphic Forms, Memoirs of the American Mathematical Society, no. 299, American Mathematical Society, Providence, RI, 1984. MR.0743544 (86f:11093)

[32] _ Classical automorphic forms and hypergeometric functions, J. Number Theory 28 (1988), no. 2, 219-232. MR0927661 (89b:11037)

[33] H. A. Verrill, Some congruences related to modular forms, Preprint MPI-1999-26, MaxPlanck-Institut für Mathematik, Bonn, Germany, 1999.

Departments of Mathematics and Physics, University of Arizona, Tucson, Arizona 85721

E-mail address: rsm@math.arizona.edu

$U R L:$ http://www.math.arizona.edu/ ${ }^{\mathrm{rsm}}$ 